\title{
VARIABILITY AND STAR FORMATION IN LEO T, THE LOWEST LUMINOSITY STAR-FORMING GALAXY KNOWN TODAY*
}

\author{
Gisella Clementini ${ }^{1}$, Michele Cignoni $^{1,2}$, Rodrigo Contreras Ramos $^{1,2}$, Luciana Federici $^{1}$, Vincenzo Ripepi $^{3}$, \\ Marcella Marconi ${ }^{3}$, Monica Tosi ${ }^{1}$, and Ilaria Musella ${ }^{3}$ \\ ${ }^{1}$ INAF, Osservatorio Astronomico di Bologna, I-40127 Bologna, Italy; gisella.clementini@oabo.inaf.it, rodrigo.contreras@oabo.inaf.it, \\ luciana.federici@oabo.inaf.it, monica.tosi@oabo.inaf.it, michele.cignoni@unibo.it \\ ${ }^{2}$ Dipartimento di Astronomia, Università di Bologna, I-40127 Bologna, Italy \\ ${ }^{3}$ INAF, Osservatorio Astronomico di Capodimonte, I-80131 Napoli, Italy; ripepi@na.astro.it, marcella@na.astro.it, ilaria@na.astro.it \\ Received 2012 April 21; accepted 2012 July 10; published 2012 August 21
}

\begin{abstract}
We present results from the first combined study of variable stars and star formation history (SFH) of the Milky Way "ultra-faint" dwarf (UFD) galaxy Leo T, based on F606W and F814W multi-epoch archive observations obtained with the Wide Field Planetary Camera 2 on board the Hubble Space Telescope. We have detected 14 variable stars in the galaxy. They include one fundamental-mode RR Lyrae star and 11 Anomalous Cepheids with periods shorter than 1 day, thus suggesting the occurrence of multiple star formation episodes in this UFD, of which one about $10 \mathrm{Gyr}$ ago produced the RR Lyrae star. A new estimate of the distance to Leo T of $409_{-27}^{+29} \mathrm{kpc}$ (distance modulus of $23.06 \pm 0.15 \mathrm{mag}$ ) was derived from the galaxy's RR Lyrae star. Our $V, V-I$ color-magnitude diagram (CMD) of Leo $\mathrm{T}$ reaches $V \sim 29 \mathrm{mag}$ and shows features typical of a galaxy in transition between dwarf irregular and dwarf spheroidal types. A quantitative analysis of the SFH, based on the comparison of the observed $V, V-I$ CMD with the expected distribution of stars for different evolutionary scenarios, confirms that Leo $\mathrm{T}$ has a complex SFH dominated by two enhanced periods about 1.5 and 9 Gyr ago, respectively. The distribution of stars and gas shows that the galaxy has a fairly asymmetric structure.
\end{abstract}

Key words: galaxies: dwarf - galaxies: individual (Leo T) - galaxies: stellar content - stars: formation - stars: variables: Cepheids - stars: variables: RR Lyrae

Online-only material: color figures, machine-readable table

\section{INTRODUCTION}

Numerical simulations and semi-analytical models operating in the $\Lambda$-cold-dark-matter $(\Lambda$-CDM) scenario of galaxy formation (e.g., Bullock \& Johnston 2005) suggest that the halos of large spirals like the Milky Way (MW) and the Andromeda (M31) galaxies could have entirely been built up by merging of disrupted satellites. Despite an intensive search, the surviving "building blocks" of this formation process so far have remained elusive. However, new hope for the quest has been triggered in the last few years, by the discovery of many faint dwarf galaxies in the outskirts and halos of the MW and M31 spirals. The census of the new MW companions counts so far 17 newly discovered dwarf galaxies that were detected mainly from the analysis of the Sloan Digital Sky Survey (SDSS) data (see, e.g., Belokurov et al. 2007, 2010; Watkins et al. 2009; Koposov et al. 2009 , and references therein). Similarly, 12 dwarf galaxies were known to be M31 companions until 2004, of which only 6 are dwarf spheroidals (dSphs), but a wealth of 19 previously undetected new satellites were discovered in the last 5-6 years, by the panoramic surveys of the M31 halo carried out with the Isaac Newton and the Canada-France-Hawaii Telescopes (Ferguson et al. 2002; Ibata et al. 2007; McConnachie et al. 2009; Richardson et al. 2011) and, more recently, also by the SDSS (Slater et al. 2011; Bell et al. 2011).

The new dSphs are fainter than those previously known, with typical surface brightness generally around 28 mag $\operatorname{arcsec}^{-2}$ or less, hence they were named "ultra-faint" dwarfs (UFDs). With

\footnotetext{
* Based on archive data collected with the Wide Field Planetary Camera 2 on board the Hubble Space Telescope.
}

luminosities that reach as low as $10^{3} L_{\odot}$, the UFDs provide the ultimate opportunity to test models of the formation and chemical enrichment of the first bound structures, and their implications for the formation of larger galaxies (e.g., Bovill \& Ricotti 2011a, 2011b, and references therein; Tumlinson 2010). Figure 1 shows the location of classical (bright) dSphs and UFDs in the absolute magnitude versus half-light radius $\left(M_{V}-\log r_{h}\right)$ plane. The plot is an adapted and updated version of Belokurov et al. (2007, Figure 8). The MW and some of the M31 globular clusters (GCs) are also shown in the plot, for comparison, as well as the GCs of NGC 5128 and the ultracompact dwarf ellipticals in the Virgo Cluster. Likely due to an observational selection effect, the Andromeda's new satellites are generally brighter than the MW UFDs. Nevertheless, with faint luminosities typical of the bulk of GCs and large spatial dimensions typical of dSphs, both the MW and the M31 new satellites sample a totally unexplored region of the $M_{V}-\log r_{h}$ plane (see Figure 1).

The UFDs appear to be clustered in groups on the sky (see, e.g., Figure 1 of Richardson et al. 2011). Their velocity dispersions are small, $3-4 \mathrm{~km} \mathrm{~s}^{-1}$, and they are quite extended (see Figure 1). The MW UFDs seem to be particularly dark matter dominated (see Table 1 of Wolf et al. 2010, and references therein) and their chemical abundances are extreme, with large dispersions, and stars as metal poor as $[\mathrm{Fe} / \mathrm{H}]=$ -4 (see Tolstoy et al. 2009, and references therein). Often they have irregular shape or are elongated, such as Canes Venatici II, Segue I, Ursa Major II, Hercules (see Figure 5 of Belokurov et al. 2007; Figure 11 of Muñoz et al. 2010; and Figures 6 and 7 of Musella et al. 2012), which are likely distorted by the tidal interaction with the MW. Some of them (Bootes II 


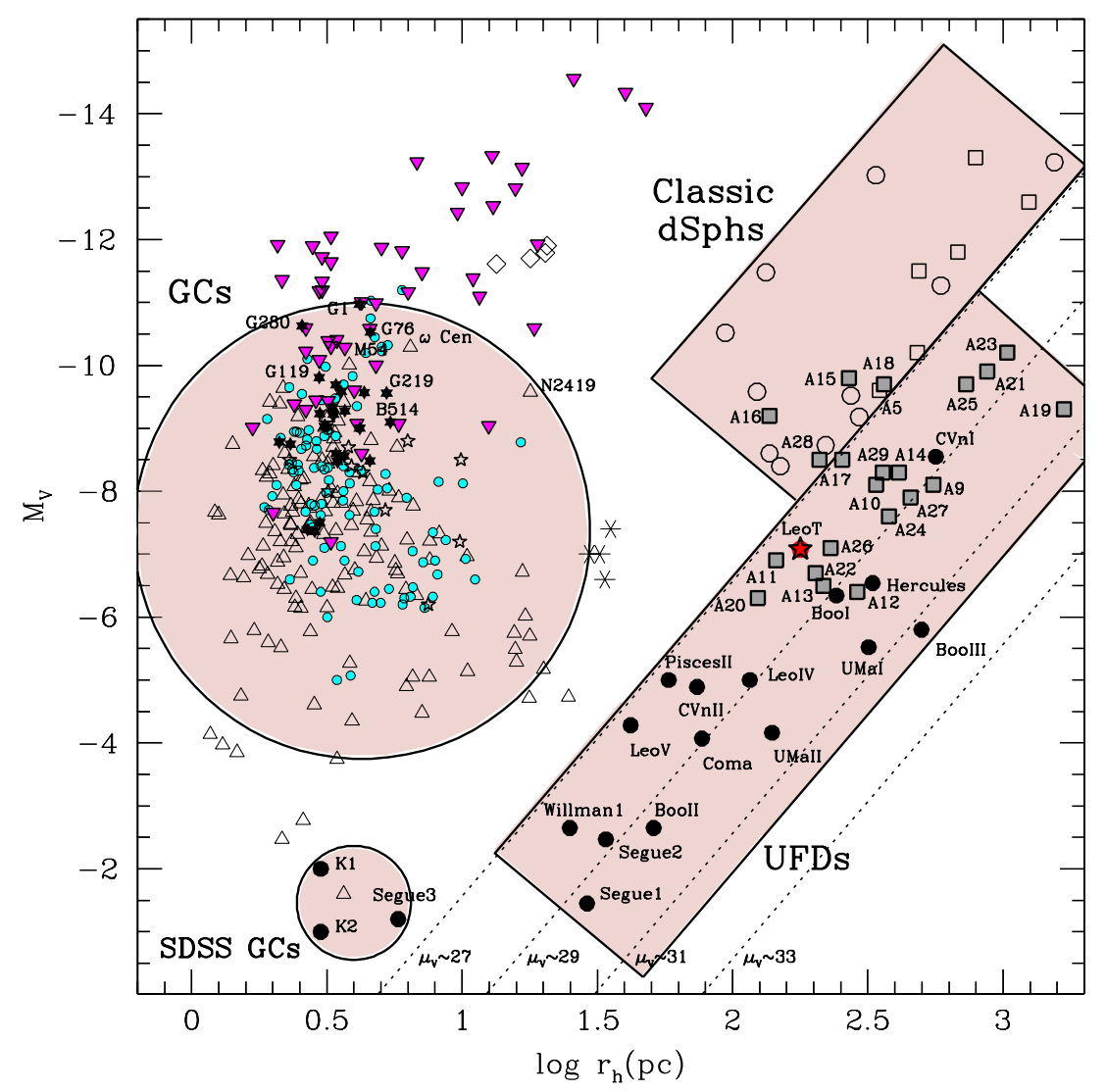

Figure 1. Absolute magnitude $\left(M_{V}\right)$ vs. half-light radius $\left(r_{h}\right)$ for different objects. Lines of constant surface brightness are marked. Open circles and squares are the classical dSphs surrounding the MW and M31, respectively. Filled circles are the MW UFDs, and three extremely low luminosity GCs, discovered mainly from the analysis of the SDSS data (see, e.g., Belokurov et al. 2007, 2010; Watkins et al. 2009; Koposov et al. 2009, and references therein). The Leo T UFD is marked by a large filled star (in red, in the online edition of the journal). Filled squares (gray) are the M31 dSph satellites discovered after 2004 (see, e.g., Richardson et al. 2011, and references therein; Slater et al. 2011; Bell et al. 2011). Open triangles are the Galactic GCs from Harris (1996) and Mackey et al. (2006). Small filled stars are the GCs of the Andromeda galaxy, from Federici et al. (2007). Small open stars are the GCs in the outer halo of M31, from Mackey et al. (2007) and Martin et al. (2006). Asterisks are the extended M31 GCs, from Huxor et al. (2005) with parameters measured by Mackey et al. (2006). Filled circles (cyan) are the GCs of NGC 5128, from McLaughlin et al. (2008). Filled inverted triangles (magenta) are the nuclei of dwarf elliptical galaxies in the Virgo Cluster, from Coté et al. (2006). Diamonds are the ultra-compact dwarfs in the Fornax cluster, from De Propris et al. (2005).

(A color version of this figure is available in the online journal.)

and Segue I) seem to be entangled in complex kinematic streams such as the Sagittarius tails (see map on V. Belokurov's Web site: http://www.ast.cam.ac.uk/ vasily/sdss/field_of_streams/ dr6/fos_dr6_marked_names.tif), see Law \& Majewski (2010) for an in-depth discussion of this issue. All UFDs host an ancient population as old as about $10 \mathrm{Gyr}$, and generally have GC-like color-magnitude diagrams (CMDs), resembling, although more dispersed than, the CMDs of metal-poor Galactic clusters like M92, M15, and M68 (Belokurov et al. 2007; Moretti et al. 2009; Musella et al. 2012; Brown et al. 2012).

The only remarkable exception to this general behavior is represented by the Leo T UFD (Irwin et al. 2007). Discovered as a stellar overdensity in the SDSS Data Release 5, the galaxy's CMD, based on follow-up observations with the $2.5 \mathrm{~m}$ Isaac Newton Telescope, revealed that Leo $\mathrm{T}$ is characterized by an intermediate-age stellar population with a metallicity of $[\mathrm{Fe} / \mathrm{H}] \sim-1.6 \mathrm{dex}$, together with a young population of blue stars of age $200 \mathrm{Myr}$ (Irwin et al. 2007). These authors estimated for the galaxy a distance modulus of $(m-M)_{0}=$ $23.1 \mathrm{mag}$ (corresponding to a distance $D=417 \mathrm{kpc}$ ), a halflight radius $r_{h}\left(\right.$ Plummer $\left.^{4}\right)=1.4$, a surface brightness $\mu_{0, V}$

\footnotetext{
4 Irwin et al.'s (2007) half-light radius is derived by fitting the radial profile
} of Leo T with a standard Plummer law (Plummer 1911).
$($ Plummer $)=26.9 \mathrm{mag} \operatorname{arcsec}^{-2}$, and an integrated magnitude $M_{\text {tot, } V}=-7.1 \mathrm{mag}$. According to these parameters the galaxy is located at the bright end of the MW UFD distribution in the $M_{V}-\log r_{h}$ plane (see Figure 1), ranking second only to Canes Venatici I (CVn I), and in the transition region between the MW and the M31 UFDs. The deeper ( $g \lesssim 26.5 \mathrm{mag}$ ) CMD published by de Jong et al. (2008) confirms the presence in Leo T of very young stars with ages between $\sim 200 \mathrm{Myr}$ and $1 \mathrm{Gyr}$, as well as an older stellar population ( $>5 \mathrm{Gyr}$ and $[\mathrm{Fe} / \mathrm{H}] \sim-1.7 \mathrm{dex}$ ). The galaxy is embedded in an $\mathrm{H}$ cloud with a heliocentric velocity of $38.6 \mathrm{~km} \mathrm{~s}^{-1}$, a velocity dispersion of $6.9 \mathrm{~km} \mathrm{~s}^{-1}$, and an estimated mass of $2.8 \times 10^{5} M_{\odot}$ (Ryan-Weber et al. 2008) or $4.3 \times 10^{5} M_{\odot}$ (Grcevich \& Putman 2009). Leo T remains, so far, the only UFD found to contain a significant amount of neutral gas. The presence of $\mathrm{H}$ I above a certain column density is often associated with star formation (SF), and indeed Leo $\mathrm{T}$ is the only UFD, and the lowest luminosity galaxy, with ongoing SF known to date. The presence of gas also suggests that Leo $\mathrm{T}$ may be affected by internal differential reddening.

Simon \& Geha (2007) obtained Keck DEIMOS spectra of 19 red giants in Leo $\mathrm{T}$ for which they estimated a mean metal abundance of $[\mathrm{Fe} / \mathrm{H}]=-2.29 \pm 0.10$ (on the Rutledge et al. 1997 metallicity scale) with a dispersion of $\sigma_{[\mathrm{Fe} / \mathrm{H}]}=0.35 \mathrm{dex}$, from the equivalent width of the $\mathrm{Ca}$ triplet absorption lines. 
Table 1

Log of the Observations

\begin{tabular}{|c|c|c|c|c|c|}
\hline Dates & $\begin{array}{c}\text { JD Interval } \\
(-2454000) \\
\text { (d) }\end{array}$ & Filter & $\begin{array}{c}\text { Exposure Length } \\
\text { (s) }\end{array}$ & $N$ & $\begin{array}{c}\text { Total Exposure } \\
\text { (s) }\end{array}$ \\
\hline 2007 Oct 21, Dec 6 & $395.17-441.08$ & F606W & 1200 & 16 & 19200 \\
\hline 2007 Oct $29-30$, Dec $8-9$ & 403.36-444.08 & F814W & 1200 & 26 & 31200 \\
\hline
\end{tabular}

Kirby et al. (2008) have re-analyzed Simon \& Geha (2007) spectroscopic material for Leo T by applying an automated spectral synthesis technique. They derived metallicities in the range of $[\mathrm{Fe} / \mathrm{H}]=-0.12$ to -3.22 dex, with an average value of $\langle[\mathrm{Fe} / \mathrm{H}]\rangle=-2.02 \pm 0.05$ dex and dispersion $\sigma_{[\mathrm{Fe} / \mathrm{H}]}=$ $0.54 \mathrm{dex}$ (where individual stellar $[\mathrm{Fe} / \mathrm{H}]$ values were first weighted by the inverse square of the errors and then averaged to obtain the mean $[\mathrm{Fe} / \mathrm{H}]$ value). This value was later revised to $\langle[\mathrm{Fe} / \mathrm{H}]\rangle=-1.99 \pm 0.05 \mathrm{dex}, \sigma_{[\mathrm{Fe} / \mathrm{H}]}=0.52 \mathrm{dex}$, by Kirby et al. (2011). The spectroscopic mean metallicities are lower than the values adopted by Irwin et al. (2007) and de Jong et al. (2008). Simon \& Geha (2007) also measured a mean stellar velocity of $38.1 \pm 2.0 \mathrm{~km} \mathrm{~s}^{-1}$ with a dispersion of $7.5 \pm$ $1.6 \mathrm{~km} \mathrm{~s}^{-1}$, in excellent agreement with the $\mathrm{HI}$ velocity and gas velocity dispersion of $6.9 \mathrm{~km} \mathrm{~s}^{-1}$ measured by Ryan-Weber et al. (2008). From the velocity dispersion Simon \& Geha (2007) infer a dark halo mass of $(8.2 \pm 3.6) \times 10^{6} M_{\odot}$ and a mass-tolight ratio of $138 \pm 71 M_{\odot} / L_{\odot, V}$ for Leo T, while from the $\mathrm{H} \mathrm{I}$ observations, Ryan-Weber et al. (2008) infer a lower limit for the total dynamical mass of $\sim 3 \times 10^{6} M_{\odot}$ and a mass-to-light ratio of $\gtrsim 56 M_{\odot} / L_{\odot, V}$.

Rocha et al. (2011) have estimated infall times $\left(t_{\text {infall }}\right)$ for the MW satellites based on the Via Lactea II cosmological simulation (Diemand et al. 2007, 2008; Kuhlen 2010). They find that Leo $\mathrm{T}$ was accreted much more recently $\left(t_{\text {infall }} \leqslant 1 \mathrm{Gyr}\right)$ than the other MW dSph and UFD satellites (see Figure 3 of Rocha et al.'s paper) and would be just falling into the MW for the first time. This could explain why Leo T managed to retain its gas and kept forming stars until a few hundred Myr ago.

As part of a project aimed at understanding the evolution of the UFDs, their connection with the MW and M31, and with the classical dwarfs and the GCs, we have already studied seven MW UFDs (Bootes I, Dall'Ora et al. 2006; CVn I, Kuehn et al. 2008; Canes Venatici II, Greco et al. 2008; Coma, Musella et al. 2009; Leo IV, Moretti et al. 2009; Ursa Major II, Dall'Ora et al. 2012; and Hercules, Musella et al. 2012), and in this paper we present results from the combined study of variable stars, star formation history (SFH), and spatial distribution of the resolved stellar populations in Leo T. The properties of the Leo $\mathrm{T}$ variable stars provide us information on the conditions at the epochs of the formation of these variables (young ages: 50-200 Myr, from short and intermediate-period Classical Cepheids-CCs; intermediate-age: 1-2 Gyr, from the Anomalous Cepheids-ACs; and old ages: $t>10 \mathrm{Gyr}$, from the RR Lyrae stars) and, combined with deep CMDs of the stars resolved on the stacked images, have allowed us to perform a quantitative analysis of the SFH that has occurred in Leo T, using the synthetic CMD method (Cignoni \& Tosi 2010, and references therein).

Observations, data reduction, and calibration of the Leo T photometry are presented in Section 2. Results on the variable stars, the catalog of light curves, and the distance to the galaxy they lead to are discussed in Section 3. The CMD of Leo T and the galaxy SFH are presented in Sections 4 and 5, respectively.
The comparison of the spatial distribution of the Leo T gaseous and stellar components is discussed in Section 6. Finally, a summary of the main results is presented in Section 7.

\section{OBSERVATIONS AND DATA REDUCTION}

The multi-epoch images of Leo $\mathrm{T}$ (R.A.(J2000) $=$ $09^{\mathrm{h}} 34^{\mathrm{m}} 53^{\mathrm{s}} .4$; decl. $(\mathrm{J} 2000)=+17^{\circ} 03^{\prime} 05^{\prime \prime}, l=214.9, b=43.7$; Irwin et al. 2007) used in this paper were retrieved from the $H u b$ ble Space Telescope (HST) archive. They were obtained with the Wide Field Planetary Camera 2 (WFPC2) from 2007 October 21 to December 9 as part of GO program 11084 (PI: D. Zucker), and consist of $16 \mathrm{~F} 606 \mathrm{~W}(V)$ and $26 \mathrm{~F} 814 \mathrm{~W}(I)$ frames, each corresponding to a $1200 \mathrm{~s}$ exposure, thus making total exposure times of 19,200 s and 31,200 s, respectively. Specifically, the 16 observations in F606W were obtained on 2007 October 21 and December 6, in two blocks of four orbits each, with a $\sim 52$ minute gap occurring between consecutive orbits in each block and each orbit filled by a pair of $1200 \mathrm{~s}$ exposures in the same filter. The $26 \mathrm{~F} 814 \mathrm{~W}$ observations were obtained in 3 blocks, on 2007 October 29-30, December 8 and 9. The first one comprised five orbits, and the other two 4 orbits each. As in the previous case, two exposures of $1200 \mathrm{~s}$ in the same filter were acquired in each orbit, and a gap of $\sim 52$ minutes was used to separate consecutive orbits. A log of the observations is provided in Table 1. Figure 2 shows the position of the four cameras of the WFPC2 on an image of Leo T published by Ryan-Weber et al. (2008) with the overlaid H I contours. The $H S T$ observations were centered on Irwin et al.'s coordinates for the galaxy. This roughly corresponds to the common vertex of the four WFPC 2 cameras. The $2 ' 6 \times 2$ ' 6 field of view (FOV) of the WFPC2 allows almost the entire region within the halflight radius of Leo $\mathrm{T}$ to be covered.

The photometric reductions of the individual pre-reduced images supplied by the STScI pipeline were done using HSTphot (Dolphin 2000). HSTphot performs point-spread function (PSF) photometry using PSFs especially tailored to the HST/WFPC2 camera and accounts for both hot pixels and cosmic rays using the information provided in each data quality image, and the comparison of multiple exposures, respectively. HSTphot also fits the sky locally around each detected source, and provides magnitudes of the measured sources corrected for charge-transfer efficiency (Dolphin 2009), and transformed to the HST flight system and the Johnson-Cousins photometric system using the transformations available at http://purcell.as.arizona.edu/wfpc2_calib/. We used the quality information provided by the HSTphot package to clean the list of detected sources, selecting for the CMD only stellar detections with valid photometry (object type flag $=1$ ) on all input images, global sharpness parameter $-0.3 \leqslant$ sharpness $\leqslant 0.3$, and $\chi^{2} \leqslant 1.1$, in each filter. The $V, V-I$ CMD of Leo T obtained with this procedure is shown in Figure 3. Typical internal errors of the combined photometry for non-variable stars at the magnitude level of the Leo $\mathrm{T}$ horizontal branch 


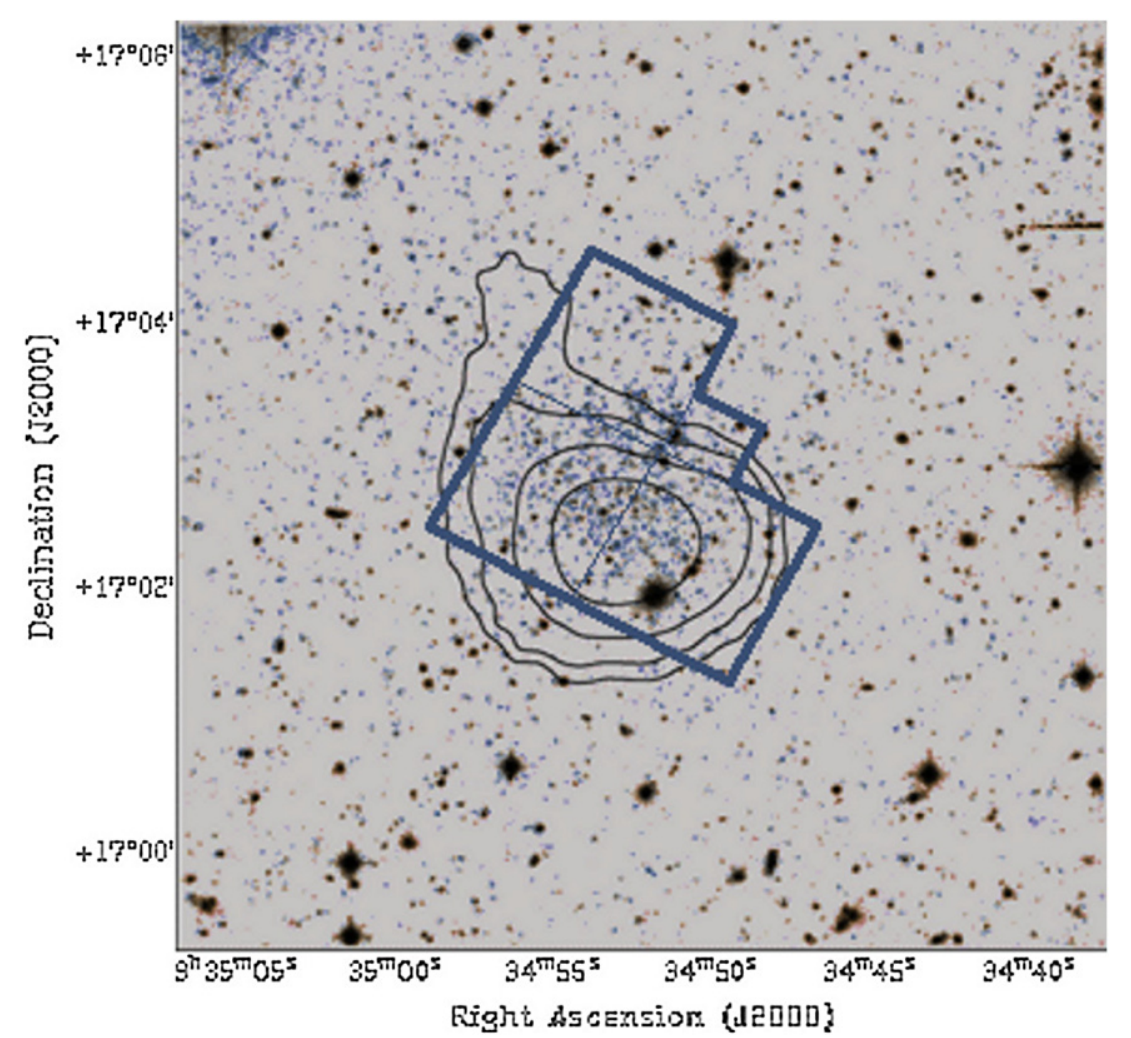

Figure 2. Map of the Leo T UFD showing the gas distribution, according to Ryan-Weber et al. (2008) and the position of the field observed with the HST. Adapted from Ryan-Weber et al. (2008, Figure 1).

(A color version of this figure is available in the online journal.)

(HB, $V \sim 23.6 \mathrm{mag}$ ) are $\sigma_{V}=0.005 \mathrm{mag}$ and $\sigma_{I}=0.006 \mathrm{mag}$, respectively.

\section{VARIABLE STARS}

The identification of the variable stars was carried out using the F606W and F814W time-series data separately, and a number of different approaches. First, candidates were identified from the scatter diagrams of the F606W and F814W data sets, using VARFIND, custom software developed at the Bologna Observatory by P. Montegriffo. The second approach was to use the Stetson variability index (Stetson 1994). Then, as a third approach and to detect potential variables fainter than the galaxy $\mathrm{HB}$, an independent search was performed on the time-series data of all sources brighter than $V=25.5 \mathrm{mag}$ ( $\sim 2500$ objects in total), which were analyzed in both filters by means of a Lomb-Scargle algorithm (Press \& Rybicki 1989) to find the most probable periods. The light curves of the candidate variables found with the above procedures were then inspected visually. Particular care was devoted to checking stars brighter than $V=22.5 \mathrm{mag}$ and with color $V-I \leqslant 0.5 \mathrm{mag}$ that, according to the comparison with the isochrones (see Section 4), might be crossing the instability strip during the blue loop (BL) phase of their evolution. At the end of the whole search process we were left with a list of about a hundred candidates, of which 14 were eventually confirmed. Final periods for these confirmed variable stars were obtained with the Grafical Analyser of Time Series (GRATIS), private software developed at the Bologna Observatory by P. Montegriffo (see, e.g., Clementini et al. 2000), which uses both the Lomb periodogram (Lomb 1976) and the best fit of the data with a truncated Fourier series (Barning 1963). The final periods adopted to fold the light curves were those that minimize the rms scatter of the truncated Fourier series best fitting the data. The scheduling of the Leo T observations is not optimized for variability studies; nevertheless, we were able to obtain reliable light curves (particularly in the $I$ band), and periods for the variable stars accurate to the third/fourth decimal place, by iterating the period search procedure between the F606W and F814W data. The variable stars are plotted on the galaxy CMD in Figure 3 using their intensity-averaged magnitudes and colors, and different symbols for the different types of variability. We have also marked with (green) filled circles the red giant branch (RGB) stars measured spectroscopically by Kirby et al. (2008) that fall in the WFPC2 FOV. The light curves of the variable stars we have identified in Leo $\mathrm{T}$ are presented in Figure 4.

The Leo T variables include one fundamental-mode RR Lyrae star with period $P=0.6027$ day, and 13 variables about one magnitude brighter than the $\mathrm{HB}$ with periods shorter than 1 day. No candidate variables fainter than the galaxy HB could be reliably confirmed, nor was any variable star detected among the few BL objects in the galaxy's CMD, hence no CCs seem to be present in Leo $\mathrm{T}$, indicating a negligible SF rate in the last half Gyr.

The identification and the main characteristics of the Leo T variables are summarized in Table 2 . The variable stars are ordered by increasing distance from the center of the galaxy as published by Irwin et al. (2007). Columns 3 and 4 provide the right ascension and declination (J2000 epoch), respectively. The coordinates were obtained by cross-matching our sources with the SDSS catalog. The SDSS IDs of the variable stars are provided in Column 2. Columns 5-7 give the variability type (see Section 3.2 for the classification of the brighter variables), the period, and the heliocentric Julian date of maximum light. 
Table 2

Identification and Properties of the Leo T Variable Stars

\begin{tabular}{lcccccccccc}
\hline \hline Name & ID & $\begin{array}{c}\alpha \\
(2000)\end{array}$ & $\begin{array}{c}\delta \\
(2000) \\
\mathrm{b}\end{array}$ & Type & $\begin{array}{c}P \\
(\text { days })\end{array}$ & $\begin{array}{c}\text { Epoch }(\mathrm{max}) \\
(-2454000)\end{array}$ & $\begin{array}{c}\langle V\rangle \\
(\mathrm{mag})\end{array}$ & $\begin{array}{c}\langle I\rangle \\
(\mathrm{mag})\end{array}$ & $\begin{array}{c}A_{V} \\
(\mathrm{mag})\end{array}$ & $\begin{array}{c}A_{I} \\
(\mathrm{mag})\end{array}$ \\
\hline V1 & 7140 & $09: 34: 53.32$ & $+17: 03: 06.2$ & AC & 0.835 & 444.010 & $22.76:$ & 22.11 & $0.85:$ & 0.54 \\
V2 & 6441 & $09: 34: 53.37$ & $+17: 02: 49.8$ & AC & 0.7983 & 442.8650 & $22.80:$ & 22.21 & $0.63:$ & 0.36 \\
V3 & 7112 & $09: 34: 52.56$ & $+17: 02: 42.1$ & Bin? & 0.4138 & 403.430 & 22.41 & 22.01 & 0.11 & 0.11 \\
V4 & 6438 & $09: 34: 54.15$ & $+17: 02: 34.7$ & AC & 0.5447 & 444.0637 & $22.59:$ & 22.07 & $0.78:$ & 0.45 \\
V5 & 7093 & $09: 34: 51.27$ & $+17: 02: 49.9$ & AC & 0.5911 & 443.999 & 22.54 & 22.03 & 0.69 & 0.44 \\
V6 & $\ldots$ & $09: 34: 55.63^{\mathrm{c}}$ & $+17: 02: 56.5$ & RRab & 0.6027 & 402.5277 & 23.59 & 23.13 & 0.88 & 0.53 \\
V7 & 7118 & $09: 34: 52.99$ & $+17: 02: 26.3$ & Bin? & 0.3966 & 395.323 & 22.57 & 22.13 & 0.16 & 0.12 \\
V8 & 7108 & $09: 34: 52.60$ & $+17: 02: 19.8$ & AC & 0.5625 & 440.880 & $22.74:$ & $22.18:$ & 0.55 & $0.35:$ \\
V9 & $\ldots$ & $09: 34: 50.38^{c}$ & $+17: 02: 37.4$ & AC & 0.5724 & 443.912 & $22.64:$ & 22.14 & $0.61:$ & 0.47 \\
V10 & 7075 & $09: 34: 50.09$ & $+17: 02: 45.1$ & AC & 0.4849 & 442.875 & 22.55 & 22.14 & 0.60 & 0.29 \\
V11 & 7212 & $09: 34: 57.23$ & $+17: 03: 11.8$ & AC & 0.7141 & 395.025 & $22.59:$ & 21.93 & 0.50 & 0.29 \\
V12 & 6542 & $09: 34: 57.25$ & $+17: 02: 25.1$ & AC & 0.590 & 395.20 & 22.86 & 22.44 & 1.03 & 0.66 \\
V13 & 7235 & $09: 34: 58.52$ & $+17: 02: 56.8$ & AC & 0.5587 & 441.102 & $22.65:$ & 22.16 & 0.74 & 0.43 \\
V14 & 7066 & $09: 34: 50.55$ & $+17: 01: 45.7$ & AC & 0.612 & 443.945 & 22.53 & 22.00 & 0.69 & 0.43 \\
\hline
\end{tabular}

Notes.

${ }^{\text {a }}$ Last four digits of the SDSS identification number, the complete number is obtained by adding 587742061055830000 .

${ }^{b}$ R.A., decl. coordinates from the SDSS catalog.

${ }^{c}$ R.A., decl. coordinates read from the 11084_17 WFPC2 LEO T image in the HLA-STSCI.

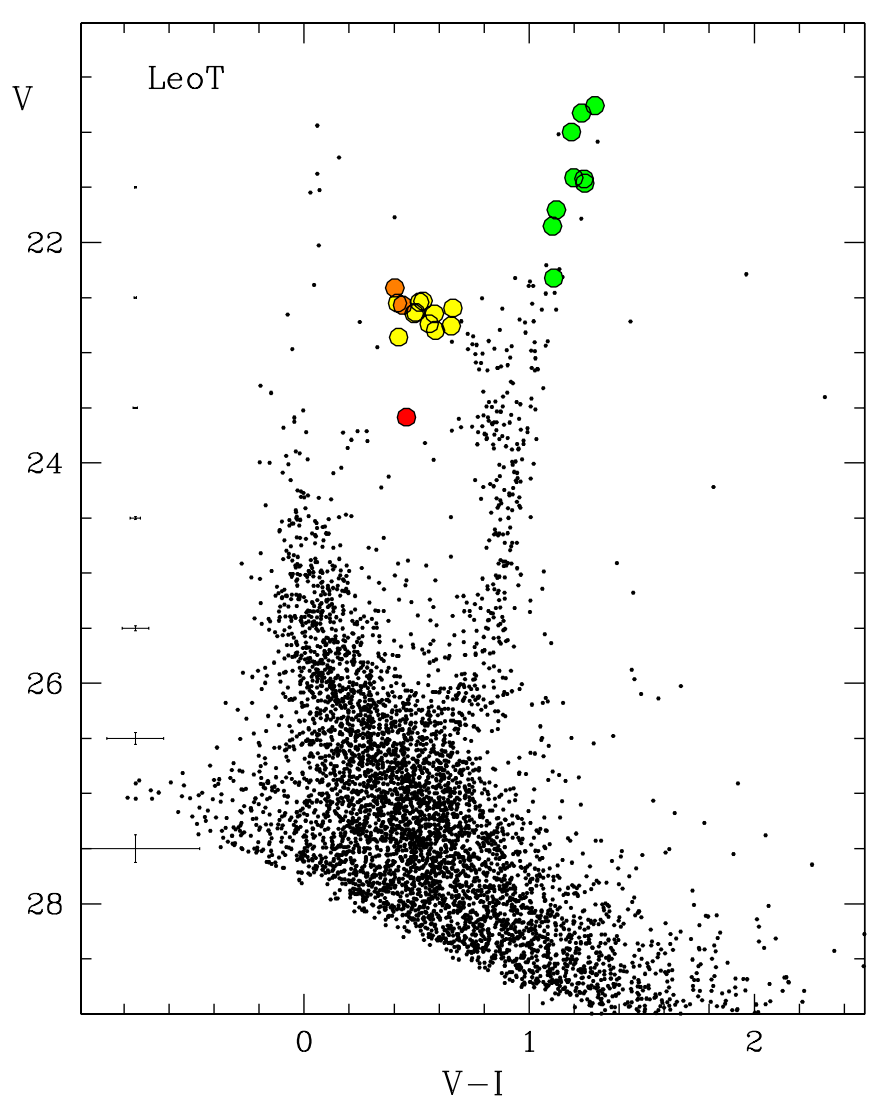

Figure 3. $V, V-I$ CMD of Leo $\mathrm{T}$ from the HST WFPC2 data. The RR Lyrae star is marked by a filled circle (in red, in the online version of the paper), the variable stars brighter than the HB by (yellow) filled circles. Among them, the (orange) filled circles are suspected binaries. The variables are plotted in the CMD according to their intensity-averaged magnitudes and colors. (Green) filled circles are Leo $\mathrm{T}$ red giant stars studied spectroscopically by Kirby et al. (2008).

(A color version of this figure is available in the online journal.)
Table 3

Johnson-Cousins $V, I$ Photometry of the Leo T Variable Stars

\begin{tabular}{lccc}
\hline \hline & \multicolumn{2}{c}{ Leo T-Star V1-AC } & \\
\hline HJD & $V$ & HJD & $I$ \\
$(-2454000)$ & $(\mathrm{mag})$ & $(-2454000)$ & $(\mathrm{mag})$ \\
\hline 395.171606 & 23.02 & 403.364745 & 22.10 \\
395.187579 & 22.97 & 403.430029 & 22.13 \\
395.237584 & 22.94 & 403.446003 & 22.20 \\
395.253558 & 22.98 & 403.496702 & 22.21 \\
395.304257 & 22.92 & 403.512676 & 22.19 \\
395.320231 & 23.03 & 403.563376 & 22.23 \\
395.370930 & 22.95 & 403.579349 & 22.25 \\
395.386903 & 22.96 & 403.630049 & 22.31 \\
440.867040 & 22.65 & 403.646022 & 22.30 \\
440.883014 & 22.74 & 442.864446 & 22.30 \\
\hline
\end{tabular}

(This table is available in its entirety in a machine-readable form in the online journal. A portion is shown here for guidance regarding its form and content.)

Columns 8 and 9 give the intensity-weighted mean $V$ and $I$ magnitudes, while Columns 10 and 11 list the amplitude in the $V$ and $I$ bands. Uncertain values for the mean magnitudes and amplitudes, due to the incompleteness of the light curves, are flagged by a colon. The Johnson-Cousins $V, I$ time-series data of the variable stars are provided in Table 3. They were obtained by calibrating the F606W and F814W data through a procedure that properly takes into account the variation in color of the variable stars during the pulsation cycle.

A finding chart of the Leo $\mathrm{T}$ variable stars is presented in Figure 5. In the map we have also marked the RGB stars measured spectroscopically by Kirby et al. (2008). The map shows that the spatial distributions of the variables, RGB stars, and gas in Leo $\mathrm{T}$ are significantly off-centered with respect to the center of the galaxy defined by Irwin et al. (2007; red cross in Figure 5). The largest fraction of galaxy stars and all the variables are confined within cameras 3 and 4 of the WFPC2. Of the variable stars, only V14 in camera 4 of the WFPC2 falls slightly outside the galaxy half-light radius. The center of the 

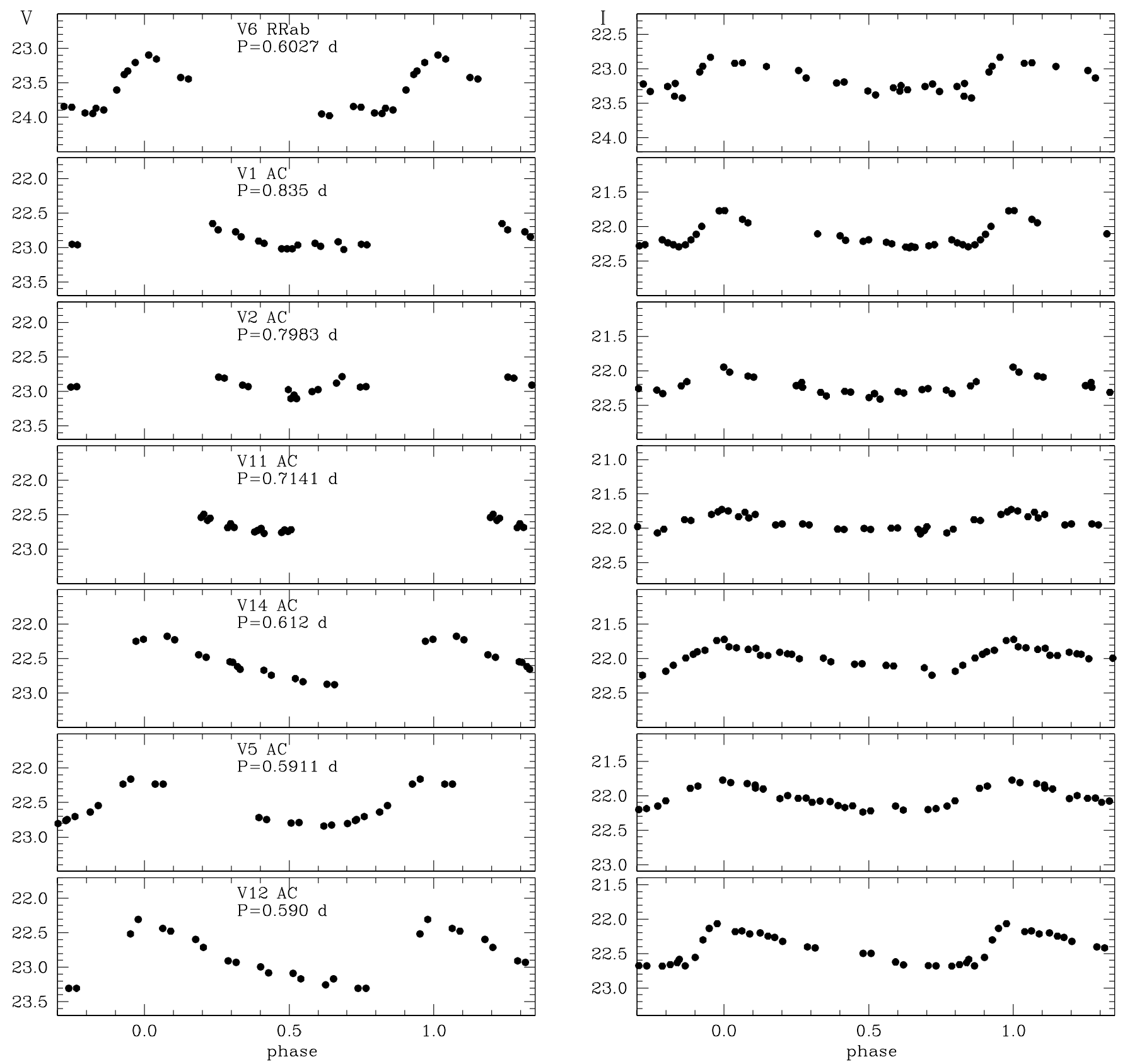

Figure 4. $V$ (left panels) and $I$ (right panels) light curves of the RR Lyrae star (top panels) and ACs identified in Leo T. Typical errors of the single data points for the RR Lyrae star are about 0.02 and $0.04 \mathrm{mag}$ in $V$ and $I$, respectively; for the brighter variables they are $0.01 \mathrm{mag}$ for $V$ in the range of 22.8-22.5 mag, and between 0.03 and $0.02 \mathrm{mag}$ for $I$ in the range of $22.4-22.0 \mathrm{mag}$.

variable star distribution seems to be in-between Irwin et al.'s center for the galaxy and Ryan-Weber et al.'s (2008) center of the $\mathrm{H}$ I distribution (blue cross in Figure 5). We will further discuss this point in Section 6.

\subsection{The Leo T RR Lyrae Star: Properties and Distance}

The period of the RR Lyrae star suggests an OosterhoffIntermediate (Oo-Int) classification ${ }^{5}$ for the Leo T UFD, with caution, though, as the classification relies on just one single

5 The Galactic GCs divide into two different groups depending on the mean period of their ab-type RR Lyrae stars: Oosterhoff type I (Oo I) clusters have $\left\langle P_{\mathrm{ab}}\right\rangle=0.55$ days, whereas type II (Oo II) clusters have $\langle P\rangle=0.65$ days. Field and cluster RR Lyrae stars in external galaxies, instead, generally have a $\left\langle P_{\mathrm{ab}}\right\rangle$ intermediate between the two Oosterhoff types; hence, they are classified as Oo-Intermediate (Oo-Int). object. We have studied so far the variable star population of other seven MW UFDs (see the Introduction section). Six of them were found to contain RR Lyrae stars with an average pulsation period resembling that observed in the Oosterhoff type II (Oo II) MW GCs (Oosterhoff 1939). The only exception was, so far, CVn I, the brightest of the MW UFDs and also the most similar to the classical dSphs, which instead appears to be Oo-Int (Kuehn et al. 2008), as also are, in fact, the vast majority of the classical dSphs (see, e.g., Clementini 2010). Leo T would thus become the second UFD showing Oo-Int properties, after CVn I.

We have derived an estimate of the distance to Leo $\mathrm{T}$ from the mean magnitude of the galaxy RR Lyrae variable (star V6). The mean magnitude of $\mathrm{V} 6$ is $\langle V\rangle=23.59 \pm 0.05 \mathrm{mag}$ (where the error takes into account both the internal error 

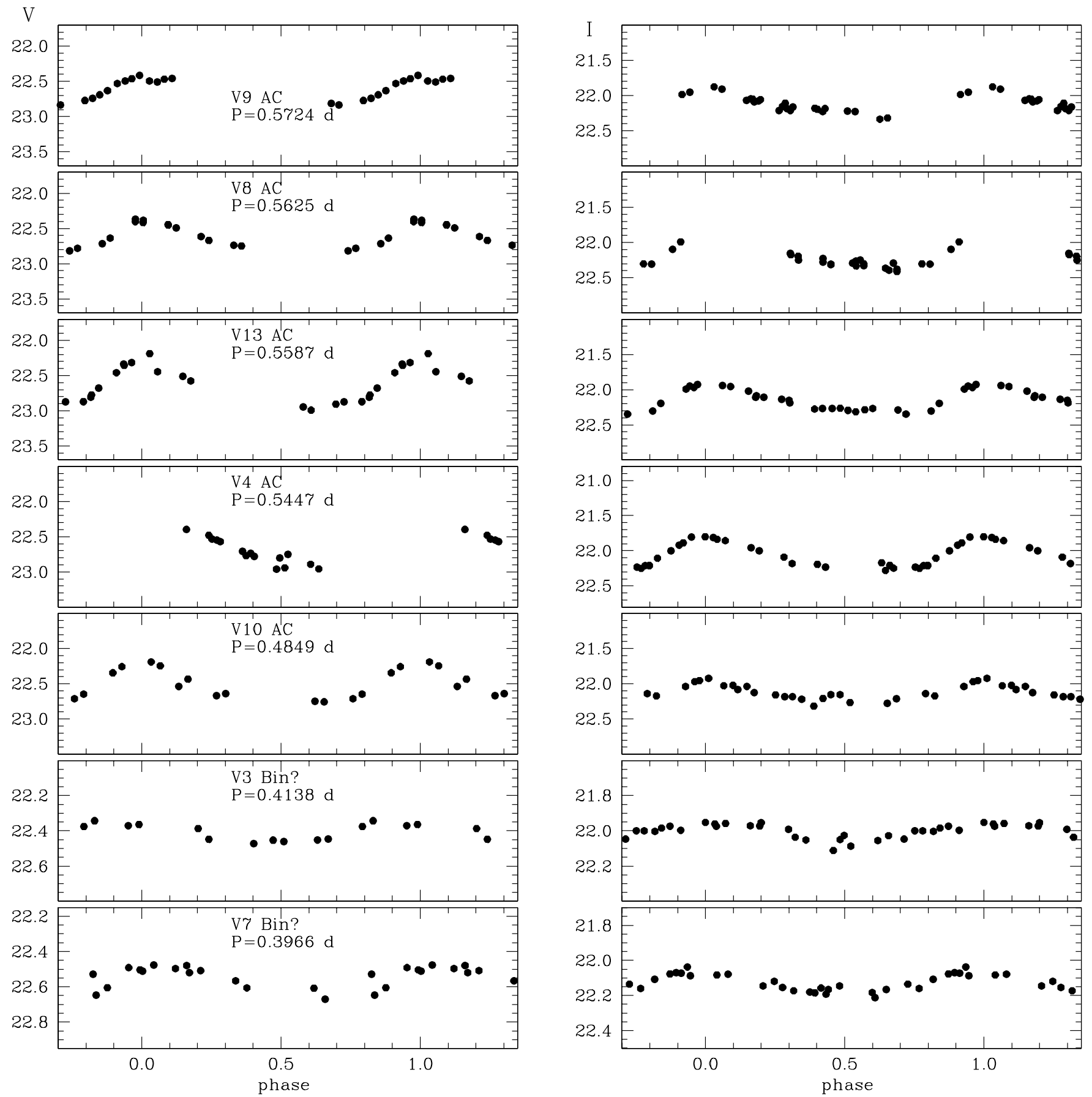

Figure 4. (Continued) The upper five pairs of panels show ACs, the lower two are suspected binaries. Note that due to the small amplitude of their light variation for the binaries we have used a $Y$-scale significantly smaller than for the ACs.

of the photometry and the uncertainty of the photometric calibration). We adopt a linear luminosity-metallicity relation for the RR Lyrae stars, with the slope value estimated by Clementini et al. (2003a) and Gratton et al. (2004), and the zero-point value consistent with a distance modulus for the LMC of $18.52 \pm 0.09 \mathrm{mag}$ (Clementini et al. 2003a), i.e., $M_{V}(\mathrm{RR})=(0.214 \pm 0.047)[\mathrm{Fe} / \mathrm{H}]+(0.86 \pm 0.09) .{ }^{6} \mathrm{Using}$ for the RR Lyrae star the mean metal abundance of the galaxy RGB

\footnotetext{
6 The zero point of the RR Lyrae luminosity-metallicity relation is still an open issue. We have adopted here the zero point of Clementini et al. (2003a), to be consistent with our latest papers on the variable stars in the UFDs, but different choices are possible (see, e.g., Cacciari \& Clementini 2003; Benedict et al. 2011).
}

stars measured spectroscopically $\langle[\mathrm{Fe} / \mathrm{H}]\rangle=-1.99 \pm 0.05$, $\sigma_{[\mathrm{Fe} / \mathrm{H}]}=0.52 \mathrm{dex}$ (Kirby et al. 2008, 2011), and a reddening value of $E(B-V)=0.031 \pm 0.026 \mathrm{mag}$, from the Schlegel et al. (1998) maps, we find a distance modulus of $23.06 \pm$ $0.15 \mathrm{mag}$ (corresponding to a distance $d=409_{-27}^{+29} \mathrm{kpc}$ ), in very good agreement with the distance modulus of 23.1 mag adopted for the galaxy by Irwin et al. (2007). Here, the errors include uncertainties in the photometry, reddening, metallicity, and in the slope and zero point of the RR Lyrae absolute magnitude versus metallicity relation. In the previous discussion we have not considered any evolution off the zero-age horizontal branch (ZAHB) of the RR Lyrae star. At such low metal abundance, evolutionary effects could make an RR Lyrae star up to 0.1 mag 


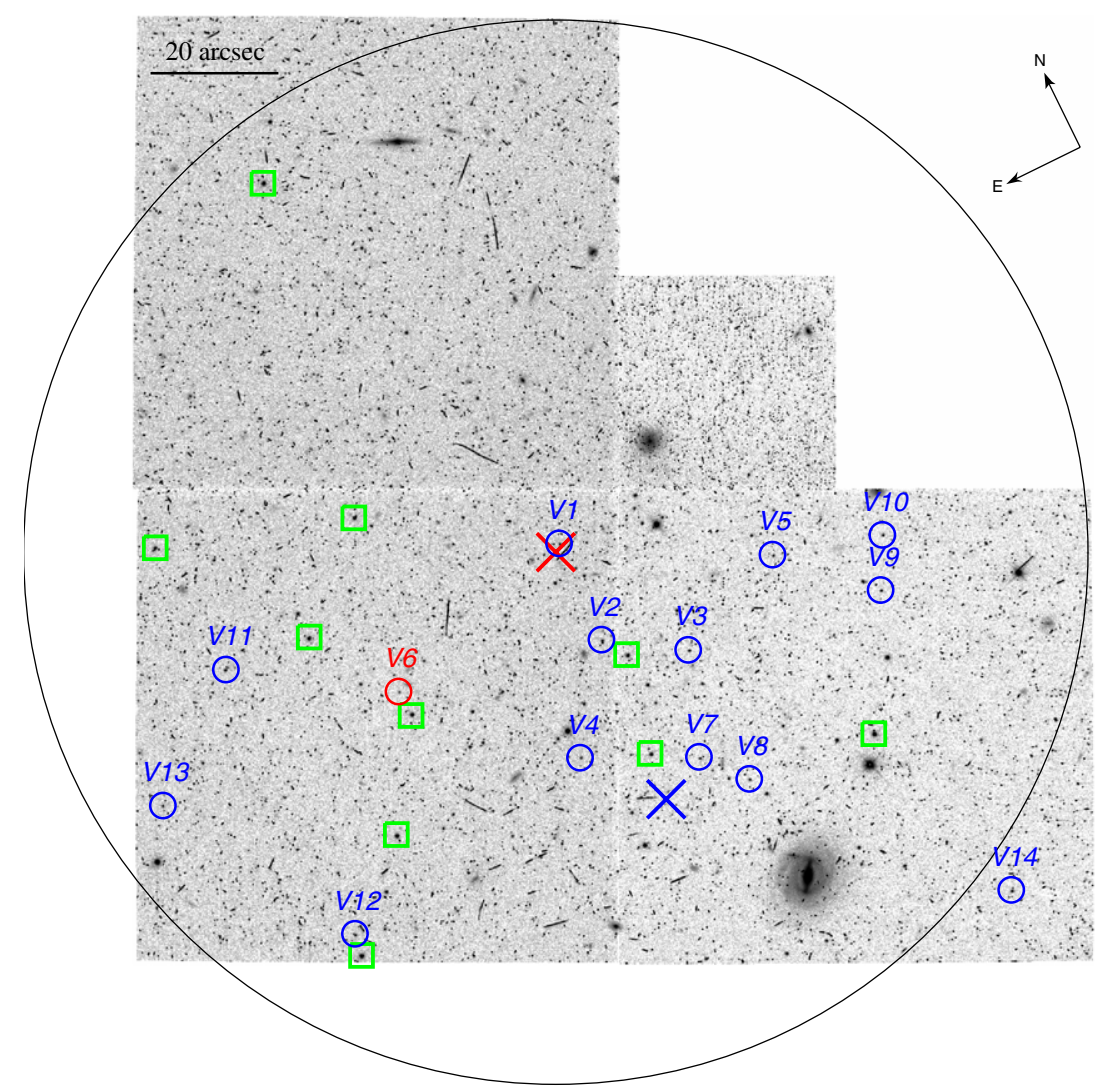

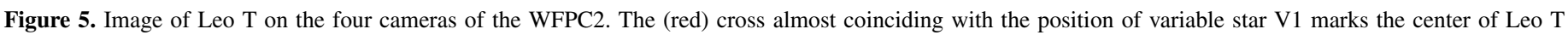

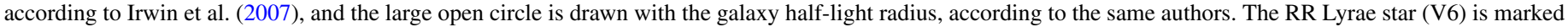

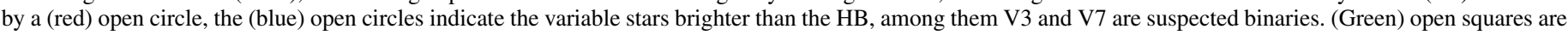
the RGB stars measured spectroscopically by Kirby et al. (2008). A (blue) cross marks the center of the H I distribution according to Ryan-Weber et al. (2008).

(A color version of this figure is available in the online journal.)

brighter than the ZAHB luminosity level (see, e.g., Caputo 1997), thus making in turn the distance modulus as long as $\sim 23.2$ mag.

\subsection{Variable Stars Brighter Than the HB: Classification, Metallicity, and Distance}

The 13 variables brighter than the RR Lyrae star are located in a region of the CMD roughly corresponding to the classical instability strip of bright pulsating stars; however, their periods and luminosities do not allow one to distinguish whether they are short-period Classical Cepheids (SPCs) or ACs. Furthermore, among them V3 and V7 have very small and nearly equal amplitudes of the $V$ and $I$ light curves, a characteristic neither observed nor expected in pulsating stars, which distinguishes V3 and V7 significantly from the other variable stars we have detected in Leo $\mathrm{T}$. We suspect that they are not pulsating variables but binaries. Unfortunately, with the present data we cannot reach a firm conclusion on the actual nature of V3 and V7, and will thus not consider them further in the following discussion on the classification of the Leo $\mathrm{T}$ bright variables.

In order to properly classify the bright variables, in the left panel of Figure 6 we have compared their position in the CMD with the ZAHB of evolutionary models for metal abundances $Z=0.0002\left([\mathrm{Fe} / \mathrm{H}]=-2.0 \mathrm{dex} ;{ }^{7}\right.$ solid line $), 0.0004([\mathrm{Fe} / \mathrm{H}]=$ $-1.7 \mathrm{dex}$; red dashed line), and $0.0006([\mathrm{Fe} / \mathrm{H}]=-1.5 \mathrm{dex}$;

\footnotetext{
7 We have adopted for the conversion from $Z$ to $[\mathrm{Fe} / \mathrm{H}]$ the simple relation:
} $[\mathrm{Fe} / \mathrm{H}]=\log Z / Z_{\odot}$, where $Z_{\odot}=0.02$. green dot-dashed line), and masses in the range of $0.65-2.0 M_{\odot}$ taken from Cariulo et al. (2004). These ZAHBs have been overplotted on the CMD assuming $E(B-V)=0.03$ mag and a distance modulus of $23.1 \mathrm{mag}$ (as adopted by Irwin et al. 2007, and in agreement with the value inferred from the RR Lyrae star). In addition, in the right panel of Figure 6 we also show the Heburning evolutionary tracks predicted for $Z=0.0002$ and masses ranging from 1.6 to $3.0 M_{\odot}$. Inspection of this plot shows that the bright pulsating variables are well reproduced by $1.6-2.0 M_{\odot}$ He-burning models originating from the ZAHB turnover (see below), and therefore having ignited the He burning in a partially degenerate core. On the other hand, the BLs of higher stellar masses (2.5 and $3.0 M_{\odot}$ in the figure) are brighter than the variables observed in Leo T. These comparisons, and the SFH presented in Section 5, seem to confirm that these variables are more likely massive HB pulsating stars, hence ACs, rather than SPCs on the BLs of a younger $(t \sim 50-200 \mathrm{Myr})$ population. Indeed, it has been suggested by several authors (Dolphin et al. 2002, 2003; Clementini et al. 2003b; Cordier et al. 2003; Marconi et al. 2004; Caputo et al. 2004; Fiorentino et al. 2006) that the ACs are the natural extension of the Population I CCs to lower metal contents and smaller masses, but see also Fiorentino \& Monelli (2012) for a recent discussion of this topic. From the evolutionary point of view, there is a general consensus that the ACs are central He-burning stars with a mass around $1.5 M_{\odot}$. Indeed, stellar evolution theory (see, e.g., Castellani \& Degl'Innocenti 1995; Caputo 1998; Fiorentino et al. 2006) has shown that for metal abundances $Z \leqslant 0.0004$ and for not-too-old 


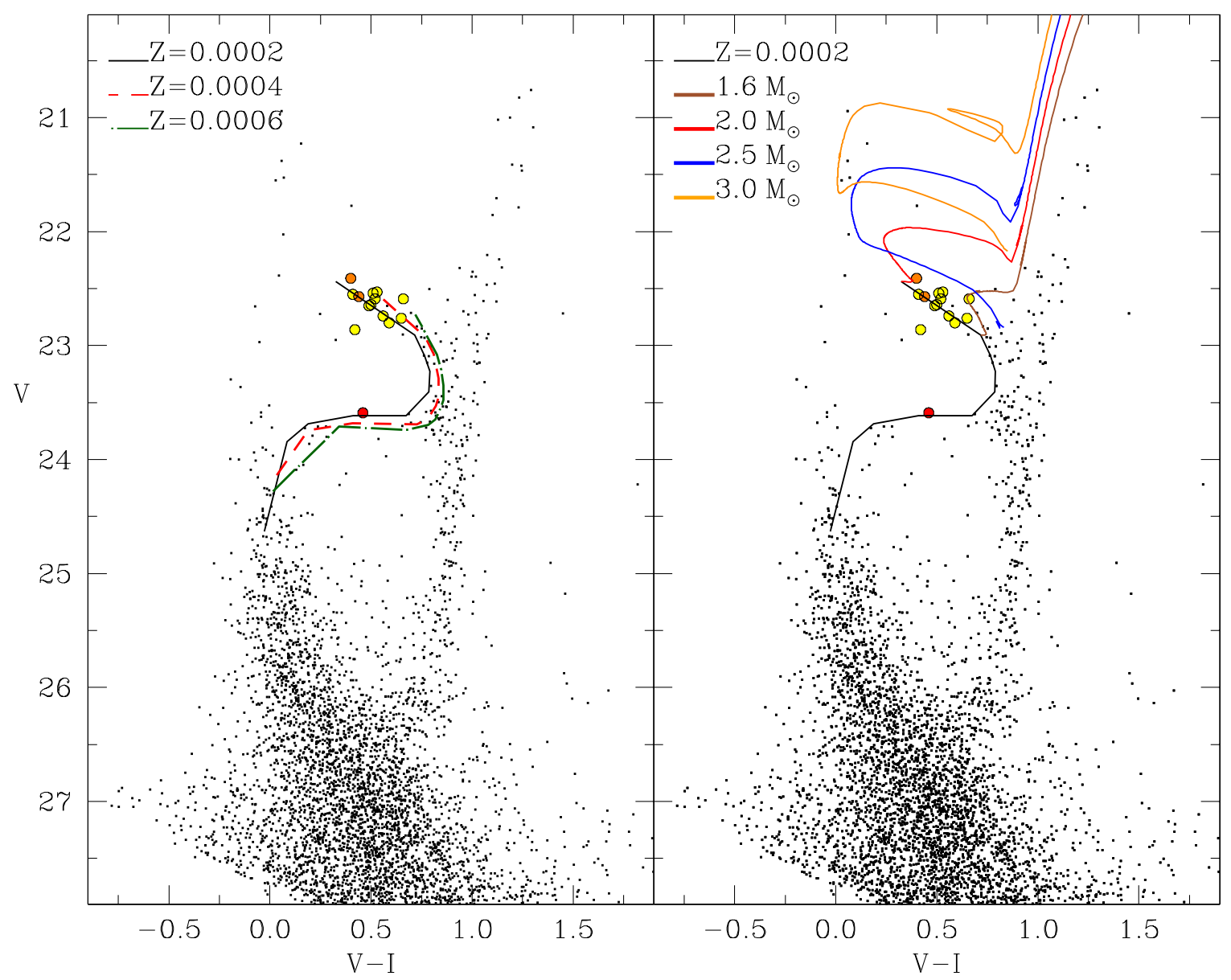

Figure 6. Left panel: $V, V-I$ CMD of Leo $T$ with superimposed the ZAHBs of evolutionary models for metal abundances $Z=0.0002$ (black solid line), 0.0004 (red dashed line), and 0.0006 (green dot-dashed line), and masses in the range of 0.65-2.0 $M_{\odot}$ from Cariulo et al. (2004; see the text for details). The ZAHBs are plotted on the observed CMD assuming $E(B-V)=0.03 \mathrm{mag}$ (from Schlegel et al. 1998) and a distance modulus of $23.1 \mathrm{mag}$, according to Irwin et al. (2007). Right panel: He burning and asymptotic giant branch evolution for stellar tracks between 1.6 and $3.0 M_{\odot}$ and metallicity $Z=0.0002$. The $Z=0.0002 \mathrm{ZAHB}$ is also shown.

(A color version of this figure is available in the online journal.)

ages ( $\leqslant 2 \mathrm{Gyr}$ ) the effective temperature in ZAHB models, which normally decreases with increasing mass, reaches a minimum at $\log T_{\mathrm{e}} \sim 3.74$ (for $Z=0.0001$ ) or $\sim 3.72$ (for $Z=0.0004$ ) around $1-1.2 M_{\odot}$. By further increasing the mass above this value, both the luminosity and the effective temperature of the ZAHB structure increase, producing a "ZAHB turnover" and an "upper HB" which intersects the instability strip again at a luminosity higher than the RR Lyrae level (see Fiorentino et al. 2006, and references therein). The effect of the higher luminosities on the periods is somehow "balanced" by the larger masses, and consequently these bright massive pulsators show periods that are not significantly longer than those typical of RR Lyrae stars, in agreement with the observed behavior for the candidate ACs in Leo T. The comparison in Figure 6 also shows that the Leo T ACs are best fitted by the $Z=0.0002$ ZAHB that very well sets the lower envelope of the distribution, while the $Z=0.0006 \mathrm{ZAHB}$ is too bright, and does not extend in color enough to reproduce the observed distribution. We also note that the difference in magnitude between the faintest massive pulsators (ACs or SPCs) and the RR Lyrae stars is predicted to be a function of metallicity (see Figure 7 in Caputo et al. 2004). Inspection of Figure 6 suggests that in the case of Leo $T$ this magnitude difference is nicely reproduced for $Z=0.0002$ with the exception of only the faintest of the bright variables, star V12.
The position of this object cannot be explained in terms of differential reddening because the reddening law would shift this object to the left of the other ACs and a significant amount of extinction would be necessary to bring it to the same luminosity level. On the other hand, if the star were more metallic $(Z \simeq$ 0.0006 ) it would not be an AC, whose predicted maximum metallicity is $Z=0.0004$, and it would be too blue to belong to the BL locus of SPCs. Furthermore, it is unlikely that V12 is a foreground RR Lyrae-type pulsator, since at the distance of $\sim 290 \mathrm{kpc}$ implied by the star's luminosity it would neither belong to Leo T nor to our own Galaxy. Perhaps, V12 is an AC-like pulsator originated from the merging of two old low mass stars. We recall that the origin of the AC pulsators is, in fact, still a matter of debate in the literature, with the two most widely accepted interpretations being that they either represent intermediate-age $(<5 \mathrm{Gyr})$ single stars produced by relatively recent SF, or that they formed from mass transfer in binary systems as old as the other stars in the parent galaxy.

As discussed in a number of theoretical papers (see, e.g., Di Criscienzo et al. 2004; Marconi et al. 2004) nonlinear convective pulsation models are able to provide firm predictions of the location of the blue boundary of the instability strip, corresponding to the blue edge of the first overtone mode. Therefore, by comparing the theoretical predictions for the metallicity of the Leo $\mathrm{T}$ candidate ACs with the observed 


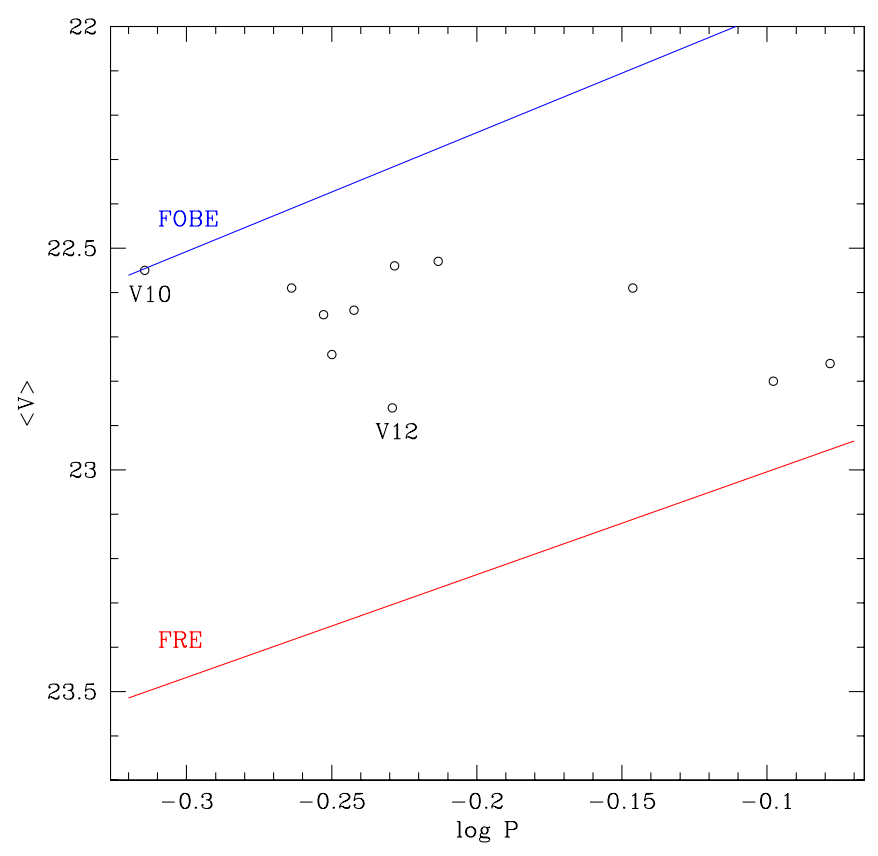

Figure 7. Comparison of the blue (FOBE) and red (FRE) boundaries of the instability strip for ACs predicted by the nonlinear convective pulsation models of Marconi et al. (2004) with the distribution of the candidate AC stars observed in Leo T.

(A color version of this figure is available in the online journal.)

distribution of these pulsators in the $M_{V}$ versus $\log P$ diagram we can derive an independent estimate of the Leo $\mathrm{T}$ distance modulus (see Caputo et al. 2000 for a detailed description of this technique). This procedure has been applied in Figure 7 where the boundaries of the AC instability strip, namely the first overtone blue edge (FOBE) and the fundamental-mode red edge $(\mathrm{FRE})^{8}$ predicted by the pulsation models of Marconi et al. (2004), have been compared with the observed distribution of the Leo T candidate AC stars on the assumption of a reddening value of $E(B-V)=0.03 \mathrm{mag}$. By fitting the position of the bluest candidate AC (star V10) to the FOBE line a good match between the theoretical and the observed blue edges is found with all the candidate ACs (open circles; as anticipated we do not considered V3 and V7 in this comparison) located well inside the theoretical strip, for a distance modulus of $23.05 \pm$ $0.10 \mathrm{mag}$, in excellent agreement with the value obtained from the Leo T RR Lyrae star. Here, the error takes into account both the uncertainty in the reddening and in the $M_{V}-\log P(\mathrm{FOBE})$ relation.

The ACs detected in Leo $\mathrm{T}$ cover a period range from $\sim 0.5$ to less than 1 day and show a spread of about 0.3 mag in visual magnitude. Pulsation models of this kind of variable (Marconi et al. 2004) suggest that near-infrared observations are needed in order to use them as standard candles through application of period-luminosity relations, and that accurate colors are needed in order to apply optical period-luminosity-color or Wesenheit relations to infer individual distances. Furthermore, these relations are predicted to be mass dependent at variance with the behavior of CCs (see Marconi et al. 2004). For these reasons we believe that further more accurate multi-band observations of Leo $\mathrm{T}$ are required in order to use these pulsators as independent distance indicators through application of the

\footnotetext{
8 This is a more uncertain prediction due to its strong dependence on the adopted treatment of convection.
}

above-mentioned relations. On the other hand, it is comforting that the comparisons of theory versus observations shown in Figures 6 and 7 provide distance moduli that are fully consistent with each other, and in very good agreement with the modulus inferred from the Leo T RR Lyrae star (see Section 3.1). Finally, we note that the presence of ACs in Leo T further strengthens the similarity with the CVn I UFD, which also contains a number of candidate ACs (Kuehn et al. 2008).

\section{THE CMD OF LEO T}

Our CMD for Leo $\mathrm{T}$ reaches $V \sim 29 \mathrm{mag}$, allowing the galaxy's main sequence (MS) to be followed well below the turn-off (TO) of the oldest stars, which is identified between $V=26.50$ and $V=27.00 \mathrm{mag}$ (see Figures 3 and 8). Several structures are clearly seen: a blue plume (BP) extending up to $V \sim 21$ mag, a slightly enlarged RGB, a clear red clump (RC) between $V \sim 23.5$ and $V \sim 23.8$ mag, a rather elongated BL, a well-defined subgiant branch (SGB), and a putative HB around $V \sim 23.7 \mathrm{mag}(\sim 23.6 \mathrm{mag}$, from the mean magnitude of the RR Lyrae star). These features reveal the presence of stellar populations of all ages. The width of the RGB may imply some chemical enrichment, as also suggested by the spread in metallicity of the RGB stars measured spectroscopically $\left(\sigma_{[\mathrm{Fe} / \mathrm{H}]}=0.52 \mathrm{dex}\right.$; Kirby et al. 2008, 20119 $)$. Signatures of youth are the bright MS and the BL phase, while the prominent $\mathrm{RC}$ is distinctive of an intermediate-age population, as also suggested by the large number of ACs detected in Leo T. Finally, the mere presence of an RR Lyrae star and the putative HB indicate stars older than 10 Gyr.

In Figure 8 we have overplotted on the Leo T CMD the Pisa isochrones (Cariulo et al. 2004) for metallicities $Z=$ $0.0002,0.0004,0.0006$ and ages $0.05,0.20,1,3,8,12$ Gyr. The adopted foreground reddening and distance modulus are $E(B-V)=0.03 \mathrm{mag}$ and $(m-M)_{0}=23.1 \mathrm{mag}$, respectively, in agreement with the distance moduli derived from the pulsating variable stars. The following conclusions can be drawn: (1) the oldest isochrones for the metallicity $Z=0.0002$ and $Z=0.0006$ bracket well the RGB width; (2) regardless of the metallicity, all models seem to (slightly) overestimate the luminosity of the $\mathrm{RC} / \mathrm{HB}$, suggesting that the adopted distance modulus sets a lower limit to the galaxy distance; (3) only a few SGB stars are located between isochrones of ages $10 \mathrm{Gyr}$ and $12 \mathrm{Gyr}$, while most of them are consistent with younger ages; and (4) above $V \sim 24 \mathrm{mag}$, only very few BP stars appear bluer than the 200 Myr old isochrones, suggesting a low SF activity in the last 200 Myr. This is consistent with the lack of CCs in Leo T. The $200 \mathrm{Myr}$ isochrone matches well the clump of stars around $V=21.5 \mathrm{mag}$ and $V-I \approx 0 \mathrm{mag}$, indicating that these stars are BL stars. These stars were checked carefully, and none of them showed a significant light variation over the time-span covered by the present data.

\section{LEO T SFH}

The determination of the full functional form of the SFH is accomplished with the population synthesis method described in Cignoni et al. (2011) and Cignoni \& Tosi (2010). The underlying stellar evolution tracks are taken from the Pisa Evolutionary Library (Cariulo et al. 2004). The core of the method consists

\footnotetext{
9 The weighted average of Kirby et al. (2008) individual metal abundances for the 10 RGB stars falling in the FOV covered by the WFPC2 observations (green filled circles in Figure 3 ) is $\langle[\mathrm{Fe} / \mathrm{H}]\rangle=-1.89$ with a dispersion of $\sigma_{[\mathrm{Fe} / \mathrm{H}]}=0.28 \mathrm{dex}$.
} 


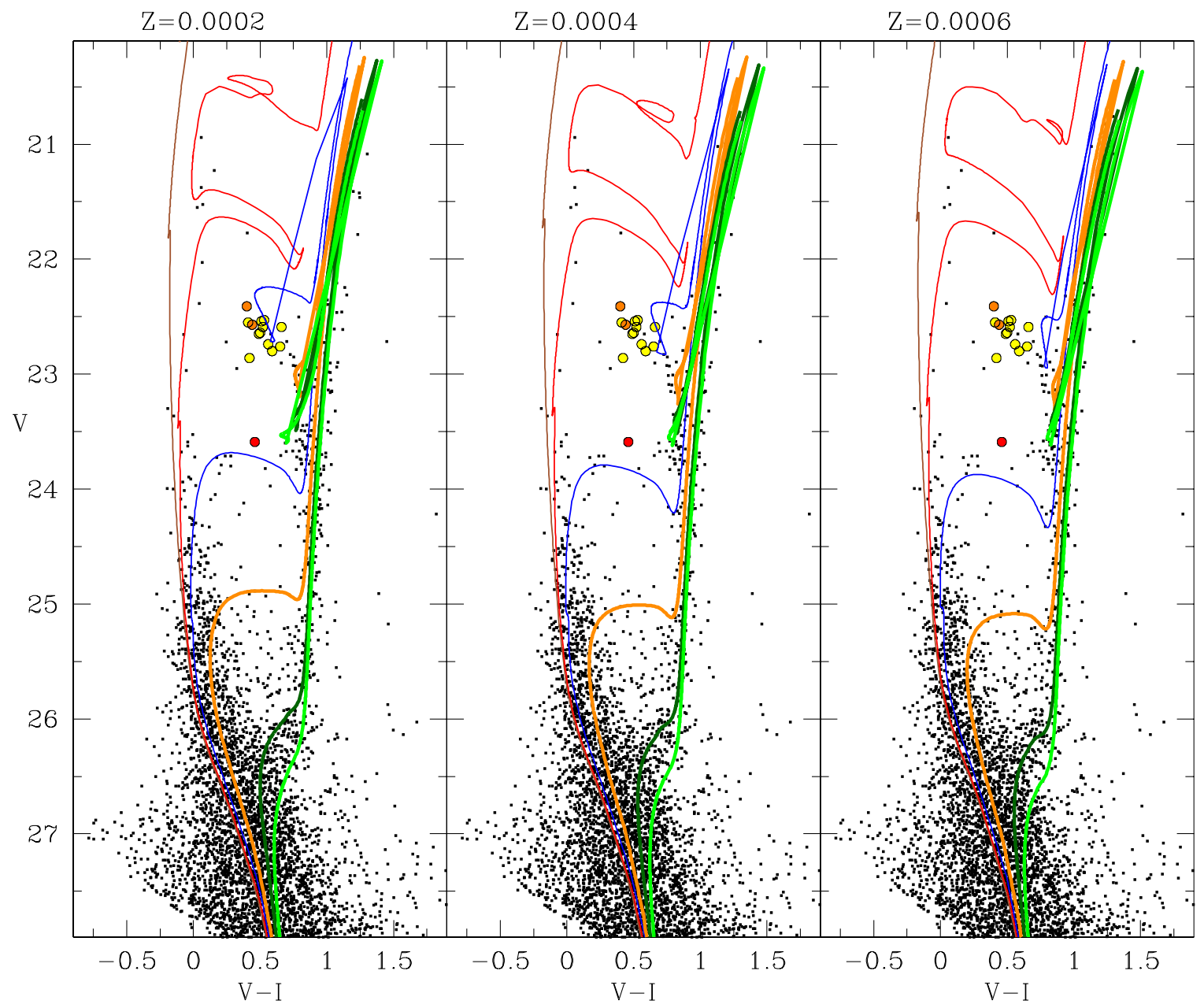

Figure 8. Observed CMD with superimposed the Pisa stellar isochrones (Cariulo et al. 2004) for metallicities (from left to right) $Z=0.0002, Z=0.0004$, and $Z=0.0006$ corrected for a distance modulus $(m-M)_{0}=23.1 \mathrm{mag}$ and a foreground reddening $E(B-V)=0.03$ mag. For each metallicity the isochrones are for populations of ages $0.05,0.2,1,3,8,12 \mathrm{Gyr}$, with the youngest isochrone being the brightest. The RR Lyrae star is marked by a (red) filled circle, the bright variables above the HB by (yellow) filled circles, the two candidate binaries by (orange) filled circles.

(A color version of this figure is available in the online journal.)

of two main steps: (1) a Monte Carlo procedure, in which a basis set of partial CMDs, characterized by a step SFH, is generated and convolved with data uncertainties as derived from extensive artificial star tests. Any model CMD is written as a linear combination of this basis; (2) a data-model comparison, in which the level of likelihood between data and model is assessed in a least-square sense. This point is addressed by minimizing a $\chi^{2}$ function of the differences in the number of stars in a set of suitable regions of the CMD. Size and morphology of the regions are motivated by the photometric errors, Poisson statistics, and stellar population involved. The optimal CMD binning scheme is found by trial and error, through several combinations of $\Delta V$ and $\Delta(V-I)$. Given the small size of the sample, a CMD grid of $0.25 \mathrm{mag}$ in $V$ and $0.1 \mathrm{mag}$ in $V-I$ was found as the most effective to keep the Poisson fluctuations low and preserve the time resolution. Once the best SFH is recovered, the statistical uncertainty is evaluated by a bootstrap technique.

To limit the number of free parameters, all synthetic CMDs were generated with (1) a Salpeter initial mass function (IMF); (2) a $30 \%$ binary fraction; ${ }^{10}$ (3) a foreground reddening of $E(B-V)=0.03 \mathrm{mag}$, according to the Schlegel et al. (1998) maps; and (4) a random metallicity between $Z=0.0002$ and $Z=0.0004$, motivated by the observed magnitude difference

\footnotetext{
${ }_{10}$ Primary and secondary masses are randomly extracted from the same IMF.
}

between ACs and the RR Lyrae stars. As additional free parameters, the distance modulus and the differential reddening $E(B-V)$ were varied in the ranges of $23.00-23.2 \mathrm{mag}$ and $0.0-0.05 \mathrm{mag}$, respectively.

The right panel of Figure 9 shows the best reconstructed SFH. The large error bars are partly due to the sample size and partly due to the photometric errors. The general trend can be summarized as follows.

1. Leo $\mathrm{T}$ has produced stars continuously since the earliest epochs with a gasping regime.

2. Two broad peaks of SF are recognizable around $1.5 \mathrm{Gyr}$ and 9 Gyr ago; approximately $36 \%$ of the stars were formed in the last 6 Gyr.

3. Three significant gaps are visible in the age range of $0.25-0.5$ Gyr, 4 Gyr to 6 Gyr, and 9 Gyr to 13 Gyr ago.

4. A low intensity SF activity started less than $250 \mathrm{Myr}$ ago.

The best-fit distance modulus is $(m-M)_{0}=23.16 \mathrm{mag}$, while the differential reddening is $\Delta(B-V)=0.02 \mathrm{mag}$.

Figure 9 shows also the corresponding best synthetic CMD (middle panel) together with the observational CMD (left panel). MS counts and shape, SGB luminosity spread and RGB color spread are found to be in excellent quantitative agreement. A difference is seen in the location of the synthetic RC, which is somewhat brighter than observed, suggesting that the 

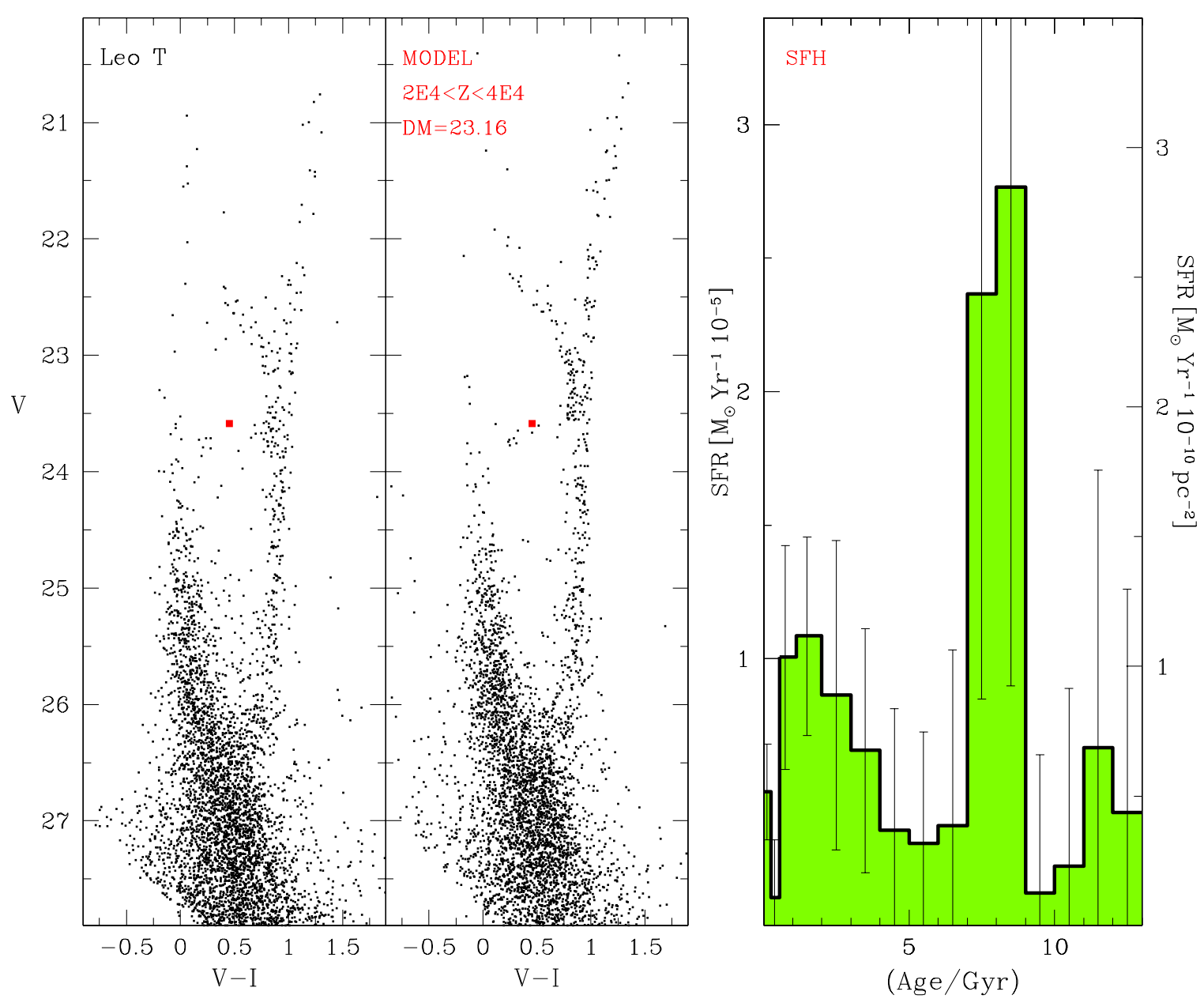

Figure 9. Left panel: observational CMD. Middle panel: best synthetic CMD. Right panel: best recovered SFH using a distance modulus $(m-M)_{0}=23.16$ mag, and $Z$ values in the range of $0.0002-0.0004$ (see the text for details).

(A color version of this figure is available in the online journal.)

average metallicity of these stars may be slightly higher than in the model. However, increasing the metallicity will ultimately reduce the magnitude difference between the ACs and the RR Lyrae star and make it lower than observed. Another issue is the paucity of synthetic BP stars above $V \approx 23 \mathrm{mag}$, a CMD region populated by massive and intermediate-mass stars on the MS and, in the most metal-poor case, also by brighter BL stars. A contamination of foreground stars is ruled out by the lack of blue stars above $g=24$ mag in the control field of de Jong et al. (2008, Figure 1); therefore this discrepancy indicates that the SF in the most recent bin (0-250 Myr ago) may be taking place on timescales shorter than the bin duration (which is imposed by photometric errors and small number statistics).

To test the robustness of our finding we have re-derived the SFH using the shorter distance modulus $(m-M)_{0}=23.06$ inferred from the RR Lyrae analysis. Figure 10 shows the corresponding SFH (right panel), the best synthetic CMD (middle panel), and the observational CMD (left panel). This SFH is fully consistent with the first one. The only clear difference is the earliest onset of the SF activity that starts earlier for the slightly longer distance modulus preferred by the SFH recovery. The match between observational and synthetic CMDs is still acceptable, although there are differences in the predicted position of helium burning stars (HB, ZAHB turnover, and $\mathrm{RC}$ ), which are brighter than the corresponding observational counterparts (RR Lyrae star, ACs, and RC stars). In conclusion, the distance modulus which provides the best-fitting SFH is slightly larger than but fully consistent with that resulting from the RR Lyrae and the galaxy's ACs.

\subsection{Comparison with Previous SFH Studies for Leo T}

de Jong et al. (2008) presented the first study of the Leo $\mathrm{T}$ SFH, based on their Large Binocular Telescope (LBT) observations of the galaxy. More recently, Weisz et al. (2012) have presented an SFH of Leo T based on the same data set used in this paper. Our results confirm those of de Jong et al. (2008) and Weisz et al. (2012), who used similar procedures to recover the SFH from their CMDs. Like us, those authors found a large amount of SF at the earliest ages, followed by a relative decrease at intermediate ages and then a renewed activity in the last few Gyr which decreases toward the present time. Interestingly, our study also revealed a previously overlooked feature, an initial activity between 10 and 13 Gyr ago significantly lower than at later epochs. Such a result is mostly driven by the TO population and the lower envelope of the SGB, CMD features that were simply not accessible to the shallower LBT sample of de Jong et al. (2008). On the other hand, these features are clearly visible in Weisz et al.'s (2012) CMD, who used the same WFPC2 observations used here. Hence the discrepancy with Weisz et al.'s (2012) SFH may be due to differences in the technique used to estimate the SFH. For instance, Weisz et al.'s 

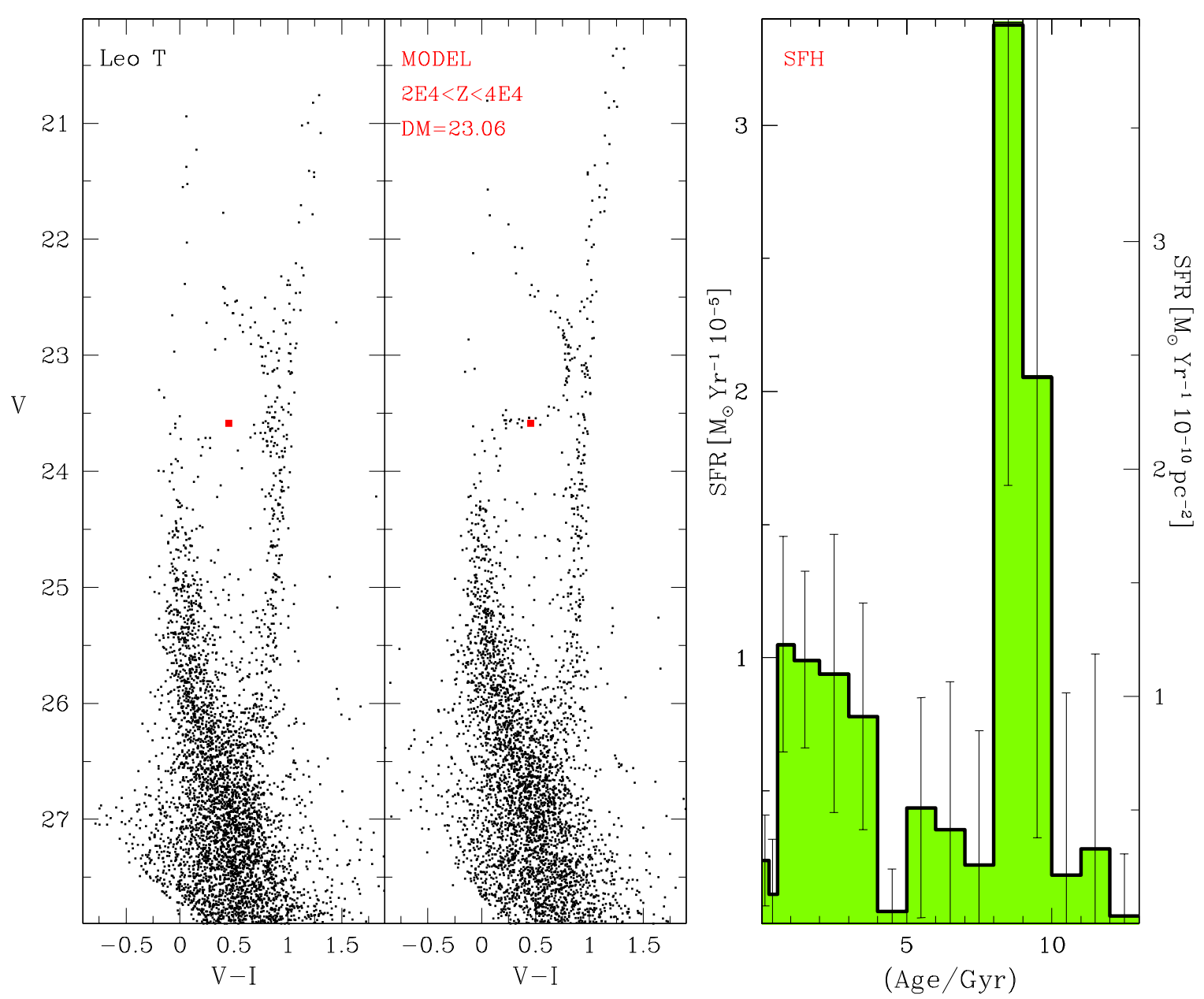

Figure 10. Left panel: observational CMD. Middle panel: best synthetic CMD. Right panel: best recovered SFH using a distance modulus $(m-M)_{0}=23.06$ mag and $Z$ values in the range of $0.0002-0.0004$.

(A color version of this figure is available in the online journal.)

(2012) distance, $(m-M)_{0}=23.05 \mathrm{mag}$, and metallicity $(Z=$ $0.0003-Z=0.0005)$ are derived simultaneously with the SFH, while in our procedure they are assumed on the basis of the variable star analysis: although our and Weisz et al.'s (2012) values are ultimately rather similar, our "a priori" assumption on the distance may have simplified the search for the best SFH.

It is also worth mentioning that (1) Weisz et al.'s preferred distance of $(m-M)_{0}=23.05 \mathrm{mag}$ is well consistent with our derived value of $(m-M)_{0}=23.16 \mathrm{mag}$, since the former was obtained for an inferred extinction of $A_{V}=0.20 \mathrm{mag}$, hence, almost a factor of two larger than ours; (2) Weizs et al.'s SFH and ours are still consistent within $1 \sigma$ errors. Indeed, $50 \%$ of Leo T's total mass was produced prior to $7.6 \mathrm{Gyr}$ ago in Weisz et al.'s solution, in good agreement with the $59 \%$ produced by our solution prior to 7 Gyr ago. Clearly, in the future it would be of great interest to explore the Leo T UFD with deeper, higher-resolution follow-up observations (HST/ACS and HST/WFC3).

\subsection{Comparison with Other Dwarf Galaxies}

Overall, and in contrast with the common view that UFDs are pure old systems, we find that Leo $\mathrm{T}$ experienced a continuous SF over most of the past 13 Gyr. In this respect the Leo T's $\mathrm{SFH}$ is a small-scaled version of the SFH occurring in brighter systems, like the dwarf spheroidals Carina and Leo I (gas free), or the dwarf irregular IC 1613 (gas rich), despite Leo T being roughly 10-100 times less massive (in terms of luminous mass). Indeed, although Leo T's stellar production is slightly peaked prior to 5 Gyr ago, Leo I and IC 1613 are both characterized by an extended event from about 5 Gyr ago until 2 Gyr ago (see, e.g., Skillman et al. 2003), a shape that is, on average, comparable with Leo T's history. Such a comparison becomes even more intriguing when we consider Carina: this galaxy experienced a minor activity prior to $12 \mathrm{Gyr}$, a dominant burst about 7 Gyr ago (that lasted about 2 Gyr), and a young burst between 2.5 and 3.5 Gyr ago (see Hurley-Keller et al. 1998). It is tantalizing to associate these episodes with the two-burst history of Leo T, keeping in mind that Leo T's bursts are spread over a longer interval of time. Moreover, while there is evidence for a moderate chemical enrichment in Leo T, Carina's bursts have different mean metallicities.

A comparison with Transition Type Dwarfs, systems which have cold gas like Leo T, but with no or very little SF, deserves a separate discussion. To date, only a couple of transition dwarf galaxies, namely Phoenix and LGS3, have been studied down to the TO of the oldest populations. Although Phoenix and LGS3 are more massive (according to Hidalgo et al. 2009, 2011 the total mass in stars is about $4.4 \times 10^{6} M_{\odot}$ and $2.0 \times 10^{6} M_{\odot}$, respectively) than Leo $\mathrm{T}$, all three galaxies are isolated members of the Local Group, so tidal interactions are not likely to have affected their evolution (which is probably driven by internal 
physical processes). Phoenix is at roughly the same distance as Leo T. The SFH recovered by Hidalgo et al. (2009) suggests that both galaxies managed to sustain the SF for almost a Hubble time. However, two major differences are noteworthy. First, the fractional mass produced by Leo $\mathrm{T}$ in the last $6 \mathrm{Gyr}(36 \%)$ is higher than in Phoenix (15\%). While feedback from supernovae may be sufficient to expel gas from their shallow potential wells, the higher mass of Phoenix may have contributed to keeping its gas density high, allowing stars to form more quickly and thus depleting the gas reservoirs. Nevertheless, if we accept this view, we are forced to conclude that dwarf galaxies like Leo $\mathrm{T}$ have probably the lowest galactic masses able to sustain the SF for a Hubble time. Second, Leo $T$ reached the maximum activity 4 Gyr later than Phoenix. Also in this case the lower mass of Leo $\mathrm{T}$ may have played a role, providing a minor shield from the UV radiation at the epoch of the re-ionization, thus delaying the SF onset.

Located at $0.65 \pm 0.05 \mathrm{Mpc}$ from us, LGS3 has an SFH (Hidalgo et al. 2011) showing similarities with both Phoenix and Leo T. Like the former it produced a larger amount of mass in the first 6-7 Gyr, followed by a low level activity which proceeded up to now. Like the latter, it experienced delayed SF relative to Phoenix: LGS3 produced the first $10 \%$ of stars 0.5 Gyr later than Phoenix, whereas Leo T produced the same percentage 2-3 Gyr later than Phoenix.

If Phoenix and LGS3 are dwarfs "in transition" between lateand early-type galaxies, Leo T may be in a different stage of this transition. This conclusion is broadly consistent with the SFH recovered by Weisz et al. (2011) for another transition dwarf galaxy, Antlia, which is even more isolated (1.3 Mpc from us) than Phoenix and LGS3. Although Weisz et al.'s CMD for Antlia does not reach the oldest MS TO, their SFH is consistent with a constant activity over the entire Hubble time.

\section{SPATIAL DISTRIBUTION OF STARS AND GAS IN LEO T}

The spatial distribution of different stellar populations in a galaxy provides a direct indication of how and where the SF took place at different times. Figure 11 shows a selection of upperMS, BL, lower-MS, and RGB stars (above and below the RC), whose spatial distributions are plotted in Figures 12 and 13. It can be seen that the distribution of the upper-MS sample (filled, red, circles in Figure 12) is similar to the BL sample (filled, blue, circles in Figure 12), with both being mostly concentrated in the WF2 camera. On the other hand, the majority of stars from the lower MS (filled, cyan, squares in Figure 12) as well as all the variable stars (ACs and RR Lyrae, marked with open, red and blue, stars, respectively) are found in the WF3 and WF4 cameras. Finally, the RGB stars are much more evenly distributed (see filled, yellow, circles and squares in Figure 13) and only slightly in excess in the WF3 and WF4 cameras.

In order to get a hint of the numerical significance of these differences, we show in Figure 14 the CMDs of stars in each of the four WFPC2 cameras, separately. As expected from the previous discussion, the camera predominantly away from the central regions (WF2) shows excess counts of BP stars above $V=24.5 \mathrm{mag}$ relative to the cameras WF3 and WF4, in spite of being globally the least populated one. This difference is particularly evident between cameras WF2 and WF4, each containing a similar number of RGB, HB, and BL stars, but rather different BP counts and shape. Moreover, the WF4's BP is rather thick and hooked around $V=24.5 \mathrm{mag}$; this is the clear signature of a dominant population 1-2 Gyr old, while the WF2's

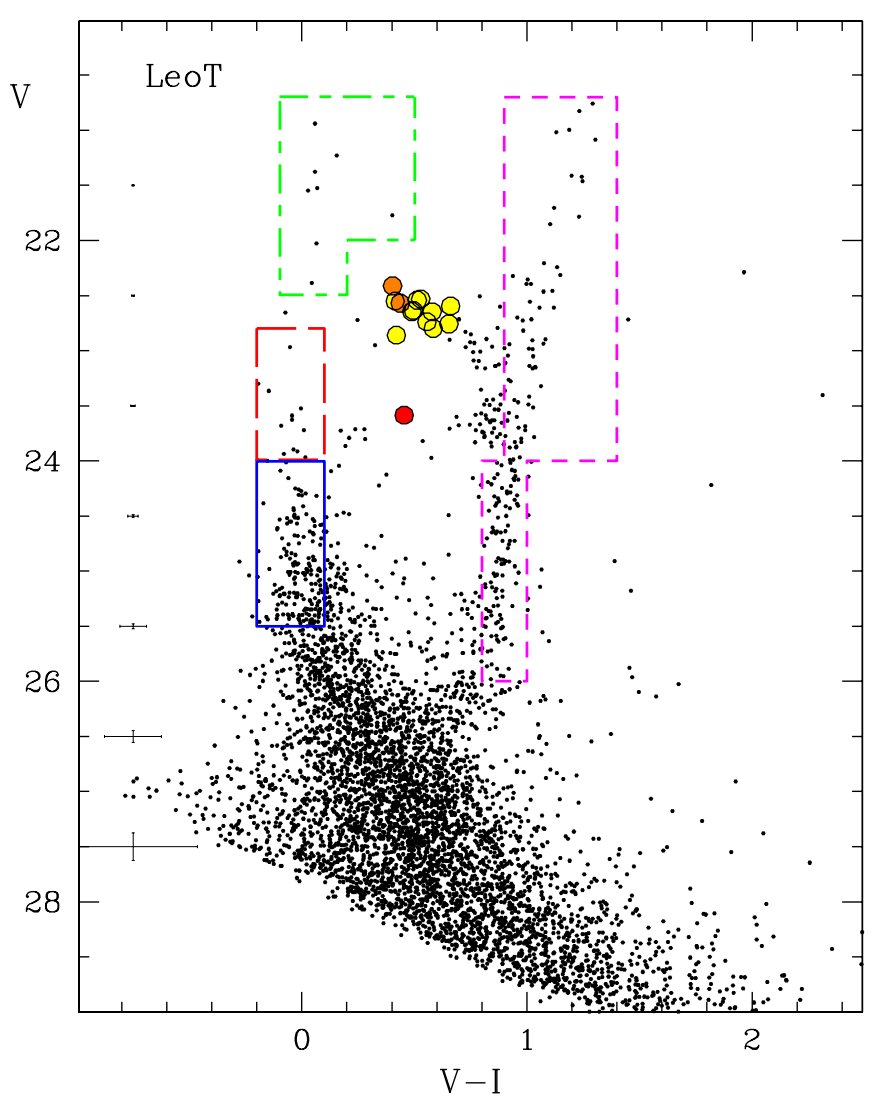

Figure 11. CMD selection of upper-MS (long-dashed, red, box), BL (dotdashed, green, box), lower-MS (solid, blue, box), and RGB stars (dashed, violet, boxes). The bona fide RR Lyrae star and the bright variables above the HB are marked by (red) and (yellow) circles, respectively, the suspected binaries by (orange) filled circles.

(A color version of this figure is available in the online journal.)

BP is sharp and extended to bright magnitudes, consistently with a population not older than 0.2 Gyr. The PC shows, instead, intermediate properties between the WF2 and WF4 cameras.

These features suggest that the young stars have a different, asymmetric distribution with respect to the bulk of older stars which trace the main body of the galaxy.

Interestingly, the H I surface density (see Figure 2) shows a similar asymmetry, characterized by a protrusion from the main (round) gas cloud. The causes of this may be manifold, ranging from an ongoing minor merger and recent accretion of external gas, to fall-down of gas that once was expelled from the galaxy in a previous starburst phase. Given the proximity of the two features (gas protrusion and excess of youngest stars in the WF2 camera) one could go a step further and suggest a recent "off-center" propagation of the SF. Enlarging the sample by including high spatially resolved observations of the Leo T's periphery would be therefore crucial and urgent to obtain a more complete picture of the galaxy's history.

\section{SUMMARY AND CONCLUSIONS}

We have studied the variable stars of the Leo T UFD and performed a quantitative analysis of the galaxy's SFH. We have detected 14 variables in Leo T, they include 1 fundamental-mode RR Lyrae star, 11 ACs, and 2 putative binaries. The average period of the RR Lyrae star $(P=0.6027$ days $)$ suggests an Oo-Int classification for Leo T, similarly only to CVn I, among the UFD galaxies. The magnitude difference between ACs and 


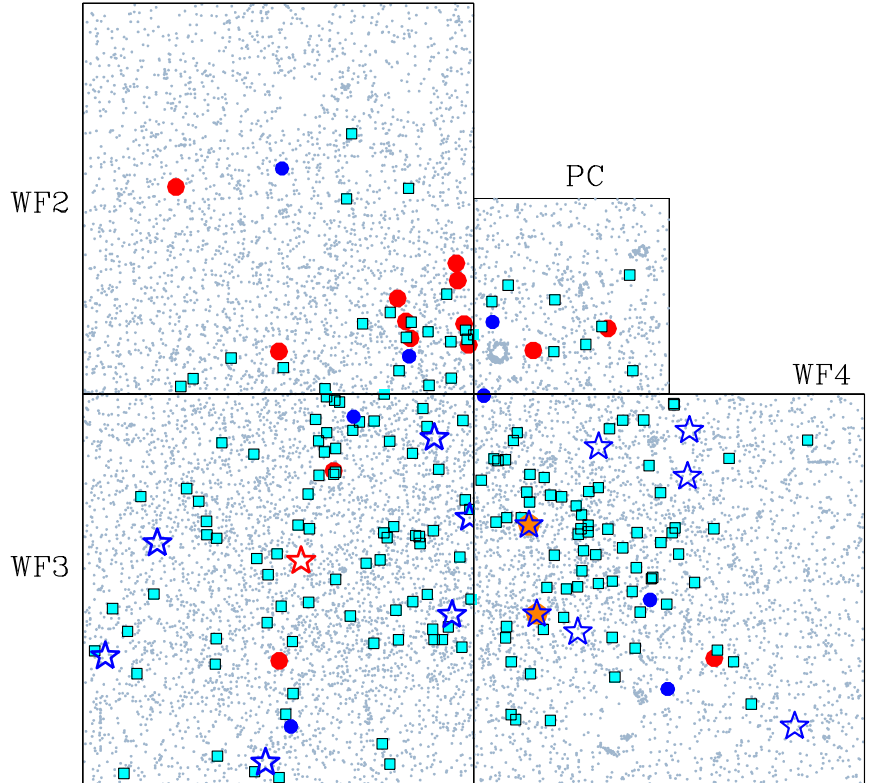

Figure 12. Spatial distribution of the Leo T stars on the 2'.6 × 2'.6 FOV covered by the four cameras of the WFPC2. (Red) filled circles mark upper-MS stars with $22.8 \mathrm{mag}<V \leqslant 24 \mathrm{mag}$, and $V-I<0.1 \mathrm{mag}$ (long-dashed, red, box selection in Figure 11); (cyan) squares are lower-MS stars with $24 \mathrm{mag}<V \leqslant 25.5 \mathrm{mag}$, and $V-I<0.1 \mathrm{mag}$ (solid, blue, box in Figure 11); (blue) filled circles are BL stars with $V<22 \mathrm{mag}$, and $V-I<0.5 \mathrm{mag}$ and $V \leqslant 22.5 \mathrm{mag}$, and $V-I<0.2$ mag (dot-dashed, green, box in Figure 11); (gray) dots show all the remaining stars. A (red) open star marks the Leo T RR Lyrae star, the (blue) open stars are the bright variables above the HB, the (orange) filled stars are the suspected binaries.

(A color version of this figure is available in the online journal.)

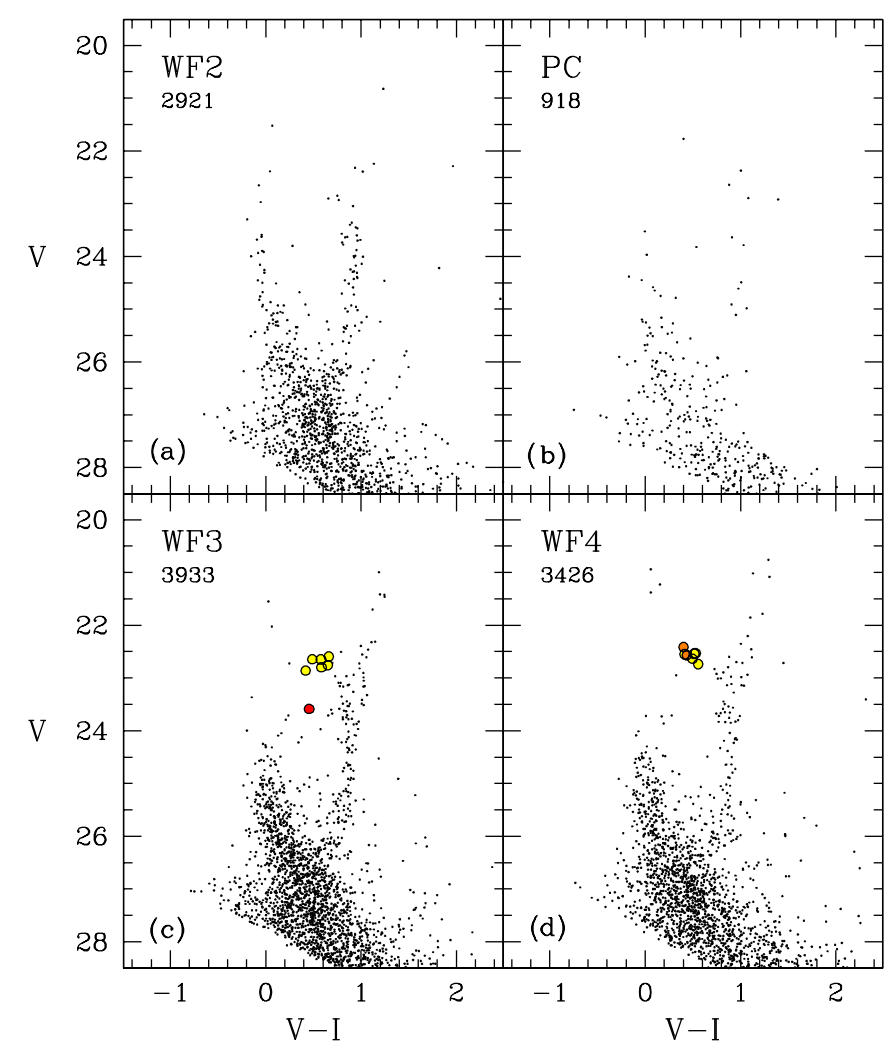

Figure 14. CMDs of Leo $\mathrm{T}$ showing (clockwise from upper left) the stellar content of the four WFPC2 cameras: WF2, PC, WF4, and WF3, respectively. The RR Lyrae star and the bright variables above the HB are marked by (red) and (yellow) filled circles, respectively, the suspected binaries by (orange) filled circles.

(A color version of this figure is available in the online journal.)

the RR Lyrae star suggests a metallicity lower than $Z=0.0004$, and more likely around $0.0002([\mathrm{Fe} / \mathrm{H}]=-2.0 \mathrm{dex})$ for the intermediate-age and the oldest stellar components in Leo $\mathrm{T}$. Adopting this metal abundance, a reddening value $E(B-V)=$ 0.03 mag and an absolute visual magnitude for the RR Lyrae star of $M_{V}(\mathrm{RR})=0.44 \mathrm{mag}($ at $[\mathrm{Fe} / \mathrm{H}]=-2.0$ dex $)$ the distance modulus inferred for Leo $\mathrm{T}$ is $(m-M)_{0}=23.06 \pm 0.15 \mathrm{mag}$, in excellent agreement with the modulus obtained by fitting the FOBE of the ACs' sample, $(m-M)_{0}=23.05 \pm 0.10 \mathrm{mag}$, and only slightly shorter than the value provided by the SFH analysis, $(m-M)_{0}=23.16 \mathrm{mag}$.

In spite of the low mass, Leo $\mathrm{T}$ underwent a complex SFH, with two major star-forming episodes, about 7-9 Gyr ago and 1-2 Gyr ago, overimposed on a continuous SF activity. The average SF rate per $\mathrm{pc}^{2}$ was around $9 \times 10^{-11} M_{\odot} \mathrm{yr}^{-1} \mathrm{pc}^{-2}$ over the whole galaxy lifetime, with a range of variation between $\approx 10^{-11} M_{\odot} \mathrm{yr}^{-1} \mathrm{pc}^{-2}$ and $\approx 4 \times 10^{-10} M_{\odot} \mathrm{yr}^{-1} \mathrm{pc}^{-2}$. Overall, these results are in good agreement with previous determinations (e.g., de Jong et al. 2008; Weisz et al. 2012), with the exception of our lower SF rate earlier than $9 \mathrm{Gyr}$ ago.

The rather isolated location, and the suggestion that the galaxy would be just falling into the MW for the first time (Rocha et al. 2011), might explain why Leo $\mathrm{T}$ managed to retain its gas and kept forming stars until 1-2 Gyr ago, for which observational evidence is provided by our identification of a conspicuous population of $\mathrm{AC}$ variables, and even later (a few hundred Myr ago), as the presence in the CMD of upper-MS and BL stars and our reconstruction of the galaxy SFH clearly demonstrate. 
Leo T's youngest stars (upper-MS and BL stars) are spatially confined in the northeast part of the galaxy (WF2 camera), whereas intermediate-age (ACs) to old populations (RR Lyrae and RGB stars) are evenly distributed across cameras WF3 and WF4, and almost absent in the WF2 camera. Similarly, the distribution of neutral hydrogen shows an asymmetric spatial distribution, with a low density protrusion extending to the northeast. The proximity of the youngest stellar component and gas protrusion suggests they are coupled, leaving us with the open question of what triggered this common anomaly in such a rather isolated UFD. Observations extending over a much larger FOV will be mandatory to address this question.

Special thanks go to Massimo Dall'Ora for turning our attention to the UFD galaxies, since the first discoveries in 2006. We warmly thank P. Montegriffo for the development and maintenance of the VARFIND and GRATIS software. Financial support for this research was provided by COFIS ASI-INAF I/016/07/0, by the agreement ASI-INAF I/009/10/0, and by PRIN INAF 2010 (PI: G. Clementini).

\section{REFERENCES}

Barning, F. J. M. 1963, Bull. Astron. Inst. Neth., 17, 22

Bell, E. F., Slater, C. T., \& Martin, N. F. 2011, ApJ, 742, L15

Belokurov, V., Walker, M. G., Evans, N. W., et al. 2010, ApJ, 712, L103

Belokurov, V., Zucker, D. B., Evans, N. W., et al. 2007, ApJ, 654, 897

Benedict, G. F., McArthur, B. E., Feast, M. W., et al. 2011, AJ, 142, 187

Bovill, M. S., \& Ricotti, M. 2011a, ApJ, 741, 17

Bovill, M. S., \& Ricotti, M. 2011b, ApJ, 741, 18

Brown, T. M., Tumlinson, J., Geha, M., et al. 2012, ApJ, 753, L21

Bullock, J. S., \& Johnston, K. V. 2005, ApJ, 635, 931

Cacciari, C., \& Clementini, G. 2003, in Stellar Candles for the Extragalactic Distance Scale, ed. D. Alloin \& W. Gieren (Berlin: Springer), 105

Caputo, F. 1997, MNRAS, 284, 994

Caputo, F. 1998, A\&AR, 9, 33

Caputo, F., Castellani, V., Degl'Innocenti, S., Fiorentino, G., \& Marconi, M. 2004, A\&A, 424, 927

Caputo, F., Castellani, V., Marconi, M., \& Ripepi, V. 2000, MNRAS, 316, 819

Cariulo, P., Degl'Innocenti, S., \& Castellani, V. 2004, A\&A, 421, 1121

Castellani, V., \& Degl'Innocenti, S. 1995, A\&A, 298, 827

Cignoni, M., \& Tosi, M. 2010, Adv. Astron., 2010, 158568

Cignoni, M., Tosi, M., Sabbi, E., Nota, A., \& Gallagher, J. S. 2011, AJ, 141, 31

Clementini, G. 2010, in Variable Stars, the Galactic Halo and Galaxy Formation, ed. C. Sterken, N. Samus, \& L. Szabados (Moscow: Sternberg Astronomical Institute of Moscow University), 107

Clementini, G., Di Tomaso, S., Di Fabrizio, L., et al. 2000, AJ, 120, 2054

Clementini, G., Gratton, R. G., Bragaglia, A., et al. 2003a, AJ, 125, 1309

Clementini, G., Held, E. V., Baldacci, L., \& Rizzi, L. 2003b, ApJ, 588, L85

Cordier, D., Goupil, M. J., \& Lebreton, Y. 2003, A\&A, 409, 491

Coté, P., Piatek, S., Ferrarese, L., et al. 2006, ApJS, 165, 57

Dall'Ora, M., Clementini, G., Kinemuchi, K., et al. 2006, ApJ, 653, L109

Dall'Ora, M., Kinemuchi, K., Ripepi, V., et al. 2012, ApJ, 752, 42

de Jong, J. T. A., Harris, J., Coleman, M. G., et al. 2008, ApJ, 680, 1112

De Propris, R., Phillipps, S., Drinkwater, M. J., et al. 2005, ApJ, 623, L105
Di Criscienzo, M., Marconi, M., \& Caputo, F. 2004, ApJ, 612, 1092

Diemand, J., Kuhlen, M., \& Madau, P. 2007, ApJ, 667, 859

Diemand, J., Kuhlen, M., Madau, P., et al. 2008, Nature, 454, 735

Dolphin, A. E. 2000, PASP, 112, 1383

Dolphin, A. E. 2009, PASP, 121, 655

Dolphin, A. E., Saha, A., Claver, J., et al. 2002, AJ, 123, 3154

Dolphin, A. E., Saha, A., Skillman, E. D., et al. 2003, AJ, 125, 1261

Federici, L., Bellazzini, M., Galleti, S., et al. 2007, A\&A, 473, 429

Ferguson, A. M., Irwin, M. J., Ibata, R. A., Lewis, G. F., \& Tanvir, N. R. 2002, AJ, 124, 1452

Fiorentino, G., Limongi, M., Caputo, F., et al. 2006, A\&A, 460, 155

Fiorentino, G., \& Monelli, M. 2012, A\&A, 504, 102

Gratton, R. G., Bragaglia, A., Clementini, G., et al. 2004, A\&A, 421, 937

Grcevich, J., \& Putman, M. E. 2009, ApJ, 696, 385

Greco, C., Dall'Ora, M., Clementini, G., et al. 2008, ApJ, 675, L73

Harris, W. E. 1996, AJ, 112, 1487

Hidalgo, S. L., Aparicio, A., Martínez-Delgado, D., \& Gallart, C. 2009, ApJ, 705,704

Hidalgo, S. L., Aparicio, A., Skillman, E., et al. 2011, ApJ, 730, 14

Hurley-Keller, D., Mateo, M., \& Nemec, J. 1998, AJ, 115, 1840

Huxor, A. P., Tanvir, N. R., Irwin, M. J., et al. 2005, MNRAS, 360, 1007

Ibata, R., Martin, N. F., Irwin, M., et al. 2007, ApJ, 671, 1591

Irwin, M. J., Belokurov, V., Evans, N. W., et al. 2007, ApJ, 656, L13

Kirby, E. N., Lanfranchi, G. A., Simon, J. D., Cohen, J. G., \& Guhathakurta, P. 2011, ApJ, 727, 78

Kirby, E. N., Simon, J. D., Geha, M., Guhathakurta, P., \& Frebel, A. 2008, ApJ, $685, \mathrm{~L} 43$

Koposov, S. E., Yoo, J., Rix, H.-W., et al. 2009, ApJ, 696, 2179

Kuehn, C., Kinemuchi, K., Ripepi, V., et al. 2008, ApJ, 674, L81

Kuhlen, M. 2010, Adv. Astron., 2010, 162083

Law, D. R., \& Majewski, S. R. 2010, ApJ, 718, 1128

Lomb, N. R. 1976, Ap\&SS, 39, 447

Mackey, A. D., Huxor, A., Ferguson, A. M. N., et al. 2006, ApJ, 653, L105

Mackey, A. D., Huxor, A., Ferguson, A. M. N., et al. 2007, ApJ, 655, L85

Marconi, M., Fiorentino, G., \& Caputo, F. 2004, A\&A, 417, 1101

Martin, N. F., Ibata, R. A., Irwin, M. J., et al. 2006, MNRAS, 371, 1983

McConnachie, A. W., Irwin, M. J., Ibata, R. A., et al. 2009, Nature, 461, 66

McLaughlin, D. E., Barmby, P., Harris, W. E., Forbes, D. A., \& Harris, G. L. H. 2008, MNRAS, 384, 563

Moretti, M. I., Dall'Ora, M., Ripepi, V., et al. 2009, ApJ, 699, L125

Muñoz, R. R., Geha, M., \& Willman, B. 2010, AJ, 140, 138

Musella, I., Ripepi, V., Clementini, G., et al. 2009, ApJ, 695, L83

Musella, I., Ripepi, V., Marconi, M., et al. 2012, ApJ, in press (arXiv:1206.4031)

Oosterhoff, P. T. 1939, The Observatory, 62, 104

Plummer, H. C. 1911, MNRAS, 71, 460

Press, W. H., \& Rybicki, G. B. 1989, ApJ, 338, 277

Richardson, J. C., Irwin, M. J., McConnachie, A. W., et al. 2011, ApJ, 732, 76

Rocha, M., Peter, A. H. G., \& Bullock, J. S. 2011, arXiv:1110.0464

Rutledge, G. A., Hesser, J. E., \& Stetson, P. B. 1997, PASP, 109, 907

Ryan-Weber, E. V., Begum, A., Oosterloo, T., et al. 2008, MNRAS, 384, 535

Schlegel, D. J., Finkbeiner, D. P., \& Davis, M. 1998, ApJ, 500, 525

Simon, J. D., \& Geha, M. 2007, ApJ, 670, 313

Skillman, E. D., Tolstoy, E., Cole, A. A., et al. 2003, ApJ, 596, 253

Slater, C. T., Bell, E. F., \& Martin, N. F. 2011, ApJ, 742, L14

Stetson, P. B. 1994, PASP, 106, 250

Tolstoy, E., Hill, V., \& Tosi, M. 2009, ARA\&A, 47, 371

Tumlinson, J. 2010, ApJ, 708, 1398

Watkins, L. L., Evans, N. W., Belokurov, V., et al. 2009, MNRAS, 398, 1757

Weisz, D. R., Dalcanton, J. J., Williams, B. F., et al. 2011, ApJ, 739, 5

Weisz, D. R., Zucker, D. B., Dolphin, A. E., et al. 2012, ApJ, 748, 88

Wolf, J., Martinez, G. D., Bullok, J. S., et al. 2010, MNRAS, 406, 1220 